\title{
ADAPTIVE BACKSTEPPING CONTROL OF ACTIVE MAGNETIC BEARINGS
}

\author{
SILU YOU \\ Bachelor of Electrical Engineering \\ Huazhong University of Science and Technology \\ July, 2007 \\ submitted in partial fulfillment of requirements for the degree \\ MASTER OF SCIENCE IN ELECTRICAL ENGINEERING \\ at the \\ CLEVELAND STATE UNIVERSITY
}

April, 2010 
This thesis has been approved

by the Department of Electrical and Computer Engineering and the College of Graduate Studies by

Thesis Committee Chairperson, Dr. Lili Dong

Department/Date

Committee Member, Dr. Daniel Simon

Department/Date

Committee Member, Dr. Wenbing Zhao

Department/Date 


\section{ACKNOWLEDGEMENTS}

By the end of my graduate study at Cleveland State University, there are some people I really want to say thanks to.

First of all, I would like to thank my supervisor, Dr. Lili Dong, for her patience and directions that help me overcome lots of difficulties. Also, my sincere gratitude goes to other professors: Dr. Daniel Simon, Dr. Wenbing Zhao and Dr. Zhiqiang, Gao. They taught me not only academic knowledge but also how to be a professional person.

I would like to thank my friends Kai Zhang, Honglei Zhang, Yao Zhang and Shuang $\mathrm{Wu}$. We have created a life-long friendship that I will never forget.

At last, I want to give my deepest thankfulness to my parents, for their selfless love and support in my life. 


\title{
ADAPTIVE BACKSTEPPING CONTROL OF ACTIVE MAGNETIC BEARINGS
}

\author{
SILU YOU
}

\begin{abstract}
A new control methodology, adaptive backstepping control $(\mathrm{ABC})$, is applied to a linearized model of an active magnetic bearing (AMB). Our control objective is to regulate the deviation of the magnetic bearing from its equilibrium position in the presence of an external disturbance. The control approach is based on adaptive backstepping control, which is a combination of a recursive Lyapunov controller and adaptive laws. In this thesis, two types of adaptive backstepping methods are used. The first method is based on full-state feedback, for which all three states in the linearized AMB model (velocity, position, and current) are used to construct the control law. The second method is adaptive observer-based backstepping control (AOBC) where only one feedback signal (position) is employed. An exponentially convergent estimator is developed for the second adaptive controller to observe other states. It is proved that the adaptive backstepping controlled AMB system is asymptotically stable around the system's equilibrium point. Simulation results demonstrate fast and stable system response. They also verify the effectiveness and robustness of the adaptive backstepping control methods against external disturbances and system parameter variations.
\end{abstract}




\section{TABLE OF CONTENTS}

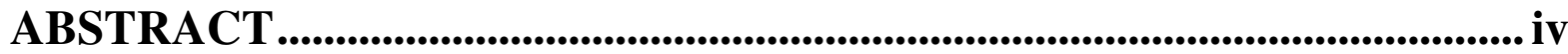

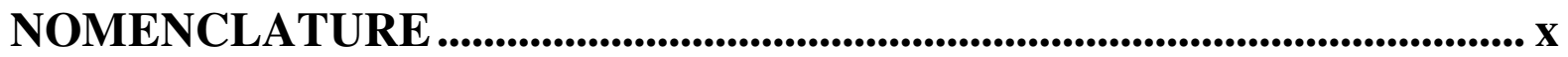

LIST OF TABLES ................................................................................................ vii

LIST OF FIGURES................................................................................................. viii

CHAPTER I INTRODUCTION ...................................................................... 1

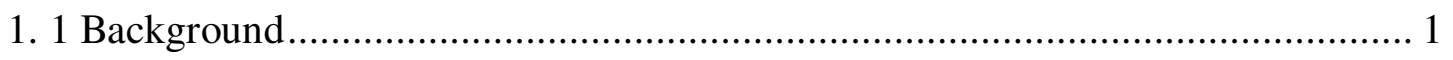

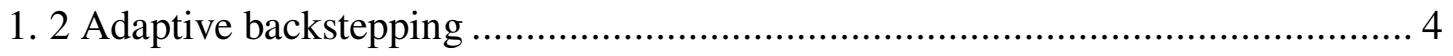

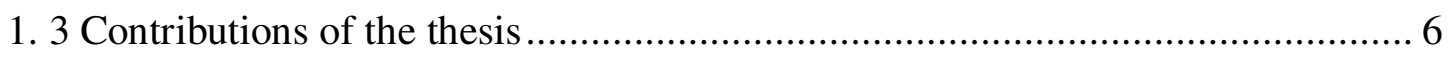

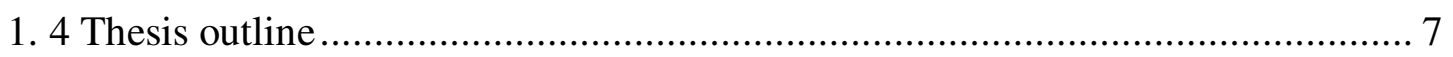

CHAPTER II MODELING OF ACTIVE MAGNETIC BEARING ........... 12

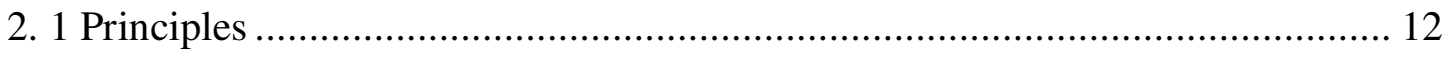

2. 2 Nonlinear model of AMB and its linearization................................................ 15

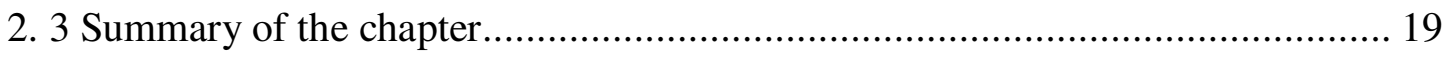

\section{CHAPTER IIIADAPTIVE BACKSTEPPING CONTROL WITH}

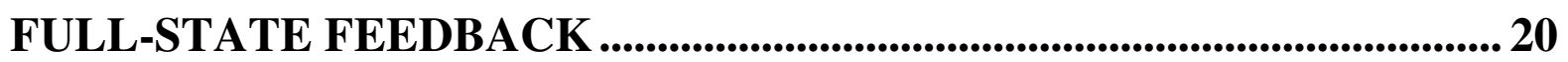

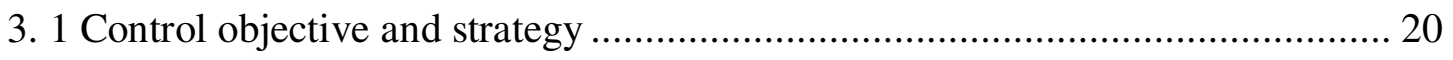

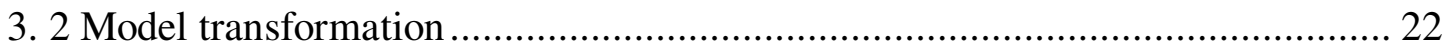




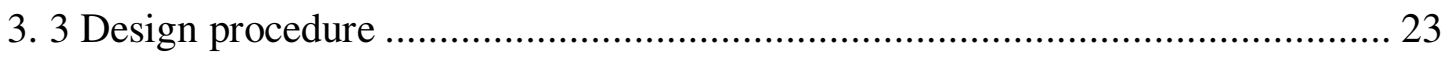

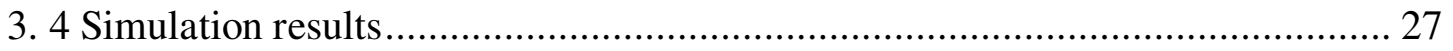

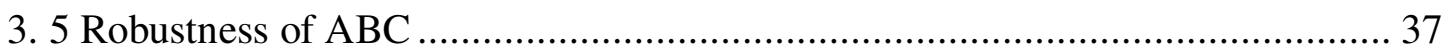

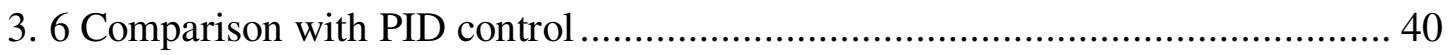

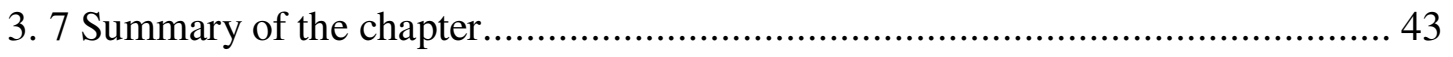

\section{CHAPTER IV_ADAPTIVE OBSERVER-BASED BACKSTEPPING}

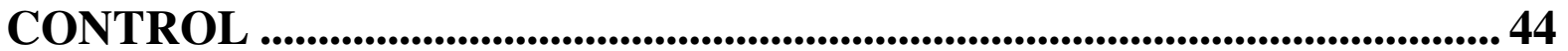

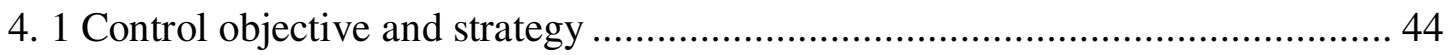

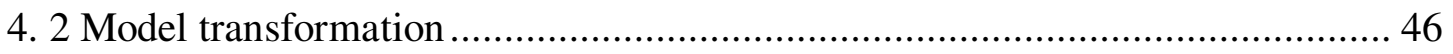

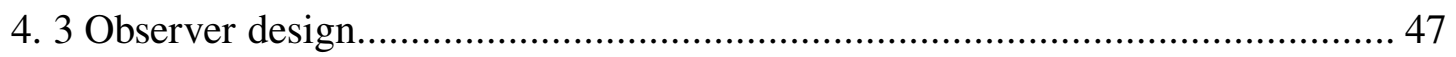

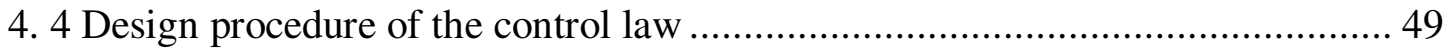

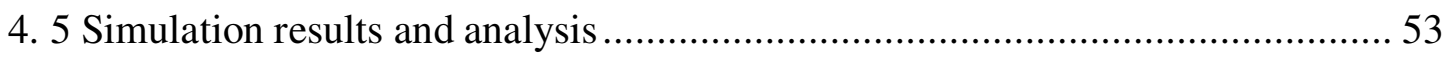

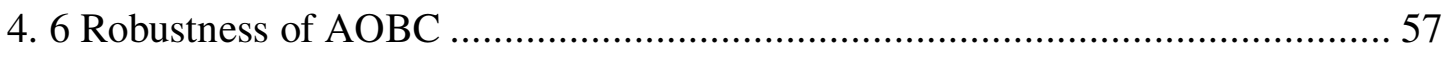

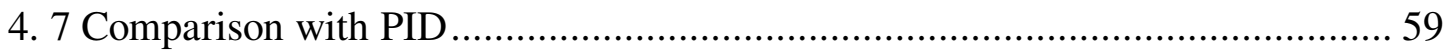

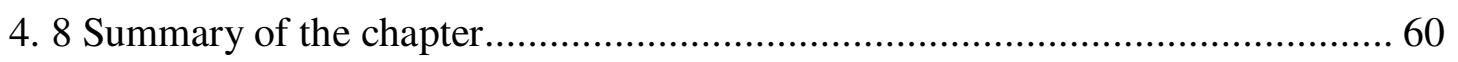

CHAPTER V_SUMMARY AND FUTURE RESEARCH .............................. 62

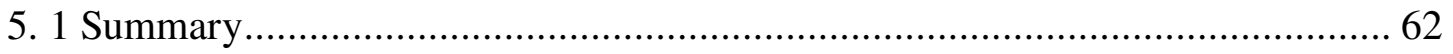

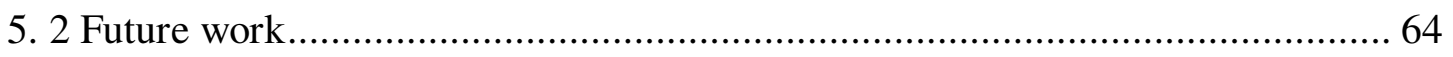

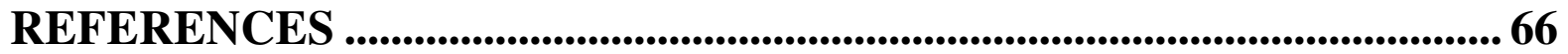




\section{LIST OF TABLES}

TABLE

PAGE

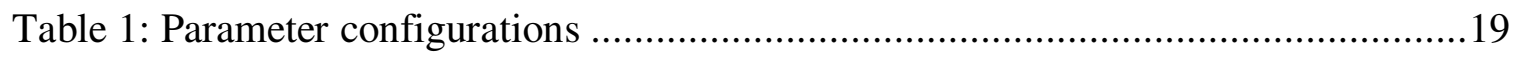

Table 2: Position of AMB with four parameters at a floating rate of $20000 \%$..................39

Table 3: Maximum factors by which system parameter can increase before the onset of

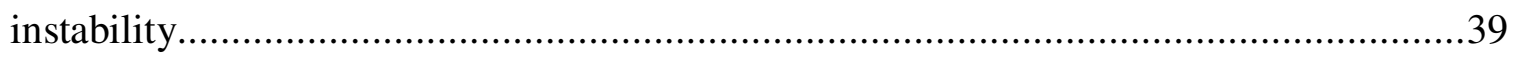

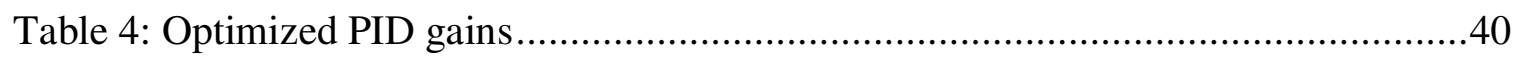

Table 5: Disturbance response characteristics in position with different Lyapunov

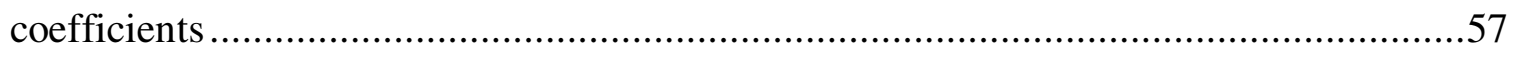




\section{LIST OF FIGURES}

FIGURE

PAGE

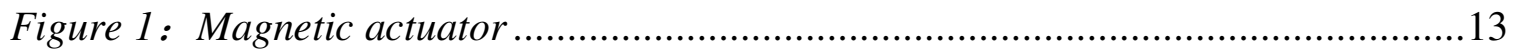

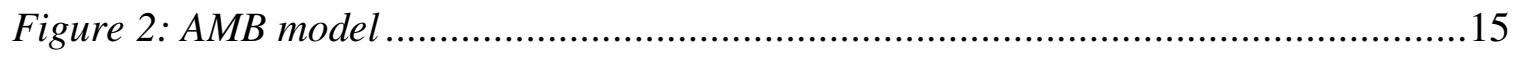

Figure 3: Block diagram of open-loop AMB plant ............................................... 18

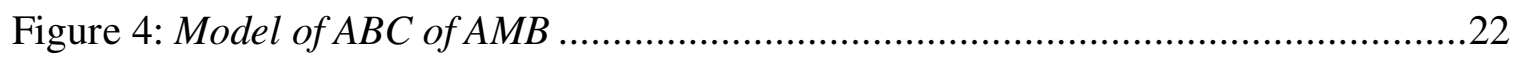

Figure 5: The closed-loop adaptive system ........................................................28

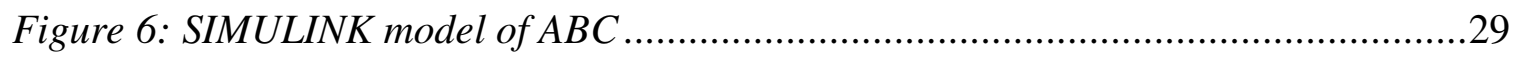

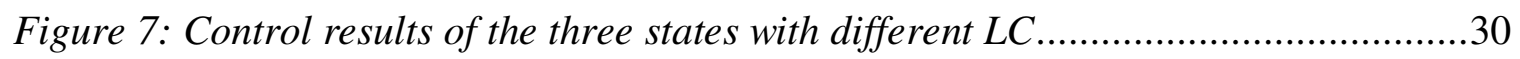

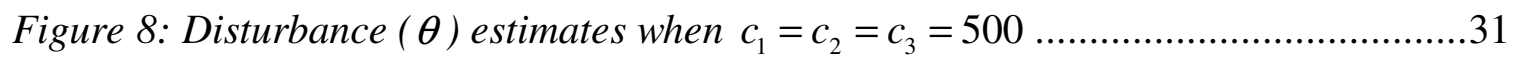

Figure 9: Disturbance and estimates when $c_{1}=3000, c_{2}=1000, c_{3}=500 \ldots \ldots \ldots \ldots \ldots \ldots . . . . .31$

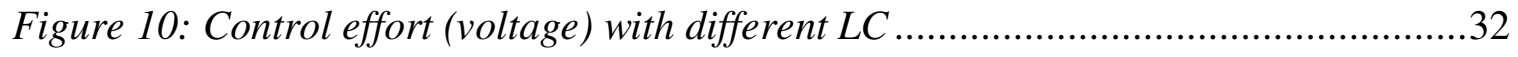

Figure 11: Control efforts with step disturbance and ramp disturbance when

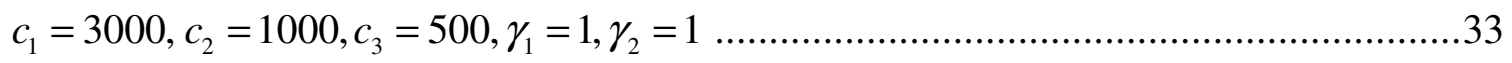

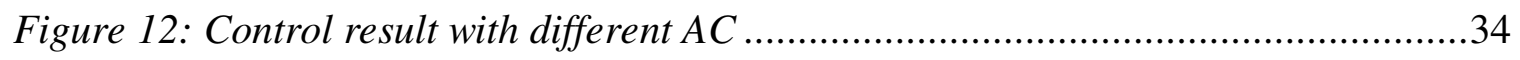

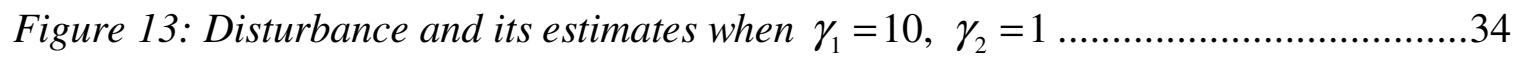

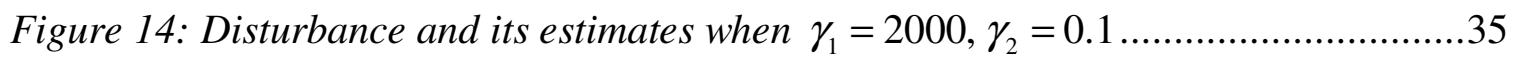

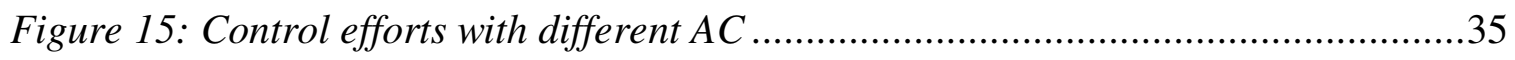

Figure 16: Position displacement when varying the parameter a to 200 and 1/200 times

its original value. .38 
Figure 17: PID control of AMB with position feedback and current control..................40

Figure 18: Position responses of $A B C$ and PID controlled AMB...............................41

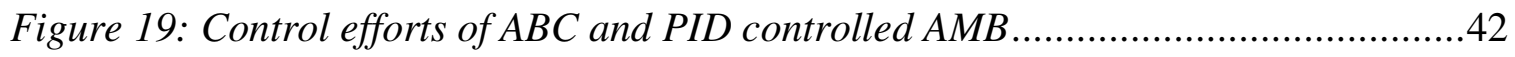

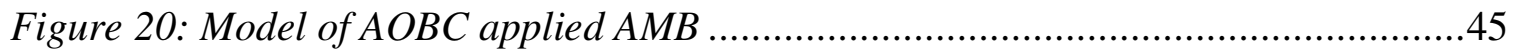

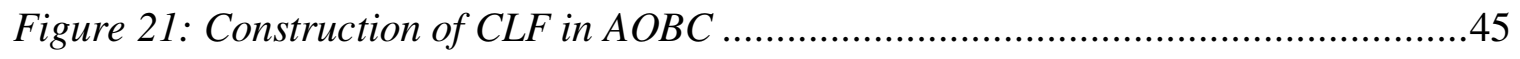

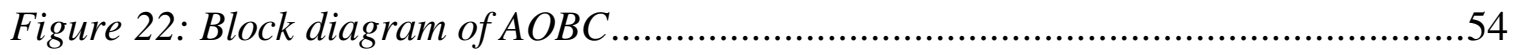

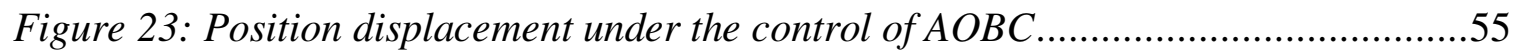

Figure 24: Disturbance estimates by three adaptive laws ..........................................55

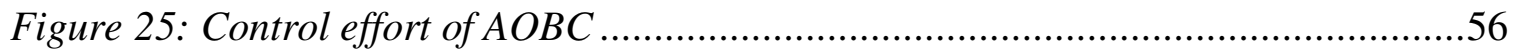

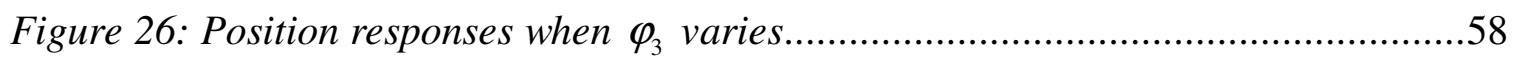

Figure 27: Position responses of $A O B C$ and PID controlled AMB systems ..................59

Figure 28: Control efforts of $A O B C$ and PID controlled AMB systems .......................60 


\section{NOMENCLATURE}

$\mathrm{ABC}$ :

AC:

AMB:

AOBC:

EMF:

CLF:

DOF:

GA:

LC:

LQR:

MIMO:

PI:

PID:

SISO:
Adaptive backstepping control

Lyapunov adaptive coefficients

Active magnetic bearing

Adaptive observer-based backstepping control

Electromotive force

Control Lyapunov function

Degree of freedom

Genetic algorithm

Lyapunov adaptive coefficients

Linear quadratic regulator

Multiple-input multiple-output

Proportional-integral

Proportional-integral-derivative

Single-input single-output 


\section{CHAPTER I}

\section{INTRODUCTION}

\section{1 Background}

Flywheel energy storage system (FESS) is an excellent alternative to chemical battery for its reliability and cost-effectiveness. It stores kinetic energy by accelerating a high speed rotor. When FESS slows down, the energy is extracted and converted back to the electricity. The amount of energy is proportional to the rotor's mass and the square of spinning speed. An important component in FESS is the magnetic bearing, which suspends the high-strength carbon-composite filaments. The magnetic bearing has two categories: passive and active ones. A passive magnetic bearing is composed of permanent magnets and the output flux can not be controlled while an active magnetic bearing (AMB) is made of electromagnets and the output flux can be adjusted by changing the current on the coil. Therefore, AMB is more popular in FESS than passive magnetic bearings. 
AMB supports the machinery using magnetic levitation produced by two opposite electromagnets [1]. Besides flywheels, it is widely used in versatile equipment such as turbo compressors, vacuum pumps and vehicle gyroscopes. AMB has several advantages compared to conventional ball or journal bearings. The most substantial advantage is that since the AMB suspends a rotor in a magnetic field, the rotor can spin at a high speed (up to $60,000 \mathrm{RPM}$ ) without contacting any mechanical part. The only friction in AMB is windage, which can be removed when AMB is operated in vacuum enclosure. This frictionless feature also leads to low-energy loss and the elimination of lubricating system [2]. In addition, $\mathrm{AMB}$ has a long life span due to the low equipment wear and its insensitive property to the pressure and temperature.

Because of its high-speed rotation and small air gap between the rotor and stator, a large deviation of rotor from its equilibrium position may trigger serious consequence which means the rotor will touch the stator, causing the failure of the operation. Therefore the control of the rotor position becomes a crucial problem. Many researchers investigated the control approaches for the AMB system. Proportional-integral-derivative (PID) control in [3]-[5] was a typical and efficient method to stabilize the rotor. Reference [3] introduced two cascade PI/PD controllers with position and current measurement values as their inputs. In [5], an estimator controller is provided to improve the quality of the damping control effort while the utilization of a single PD controller might be affected by the measurement noise. Other than PID, Linear Quadratic Regulator (LQR) control was designed and realized in a prototype small-sized AMB in [6]. References [7] and [8] 
compared several controllers such as LQR, PID, PI/PD and genetic algorithm (GA). It was discovered in [7] and [8] that the centralized controllers which took care of the entire system have better performance than the decentralized ones. However, the disadvantage of the centralized controller is being time-consuming and having computational burden. Self-sensing control of AMB was introduced in [9]-[12]. Self-sensing refers to the controller design without an extra position sensor. The position displacement thus needs to be controlled by measuring the bearing coil current. Several techniques were introduced as the compensation of the lack of the position sensor. A novel approach called Active Disturbance Rejection Control (ADRC) developed in recent years is simulated in [2] and [12], via generating a new state called "extended state" as generalized disturbance and the system response is tuned by only one parameter. Also, ADRC strategy has a high external-force-disturbance-rejection capability with the absence of position sensors just by tuning the bandwidths of controller and observer in coordination. However, as the ADRC deals with self-sensing problem, it would cause a steady-state error. But it would not be a problem as long as the error is within the bearing tolerance.

This thesis applied an adaptive backstepping method to a linearized model of the AMB and verified the effectiveness of the controller through Lyapunov method and simulation results. Adaptive backstepping control (ABC) method, developed by [13] in recent decades, is an advanced control approach associated with feedback control, Lyapunov stability theory and adaptive control. References [13]-[16] illustrated various applications of adaptive backstepping such as inverted pendulum, robot manipulator, jet engineering 
and aircraft wing rock. Several new applications were published in recent years among which [17] was about the adaptive control of helicopter attitude following desired trajectory. In [18], the time-varying speed and time-varying position commands in an induction motor drive were tracked by $\mathrm{ABC}$, and the controller was implemented using digital signal processor (DSP). The author of [18] also compared the performance of the adaptive backstepping controller with the one of PI controller and showed the superiority of the adaptive backstepping controller to PI controller. In [19] and [20], the ABC was coordinated with neural and fuzzy integral action. In [20] Gaussian Radial Basis Function Neural Network $\left(\mathrm{GRBF}_{\mathrm{NN}}\right)$ is designed to provide a full state feedback and solved the problem of both parameter uncertainty and nonlinear functions uncertainty.

In the following sections, the term $\mathrm{ABC}$ is defined as adaptive backstepping control with full-state feedback instead of the general adaptive backstepping. The AOBC is defined as adaptive observer-based backstepping control (AOBC) where only one feedback signal (position) is employed to construct the controller.

\section{2 Adaptive backstepping}

Adaptive backstepping method combines backstepping control and adaptive laws. The backstepping design starts from the first state equation where the first state variable has the highest integration order from the control input. We choose the second state variable as virtual control, and replace it with a stabilizing function [13] . This stabilizing function can stabilize the first state variable, and we set the error between virtual control and 
stabilizing function as $z$.Then for the second state equation, we will design a new stabilizing law to replace the third state variable for the new second order system [21], then "step back" to the control signal. From the steps above, we can see that the term "backstepping" means that we use the latter state as a virtual control to stabilize the previous one. This idea overcomes the shortcoming that system order can not exceed one in passive designs. Lyapunov direct method is utilized as the stabilization method for the errors between each virtual control and its stabilizing function. The control Lyapunov function (CLF) to be used will be positive definite and includes the quadratic forms of the errors.

Adaptive idea is motivated by the research of autopilot for high-performance aircrafts in 1950s [25]. In Webster, to adapt means "to change (oneself) so that one's behavior will conform to new or changed circumstances." In the control region, it could learn and tolerate the changes in system's dynamics which constant-gain feedback can not handle. The keystone of adaptive control is that a feedback controller should be able to accommodate the parameter changes by processing the output since the output signal carries the information of the system's states. Backstepping itself can not solve the uncertainty problem. However, in many systems, unknown parameters exist due to the restriction of measurement equipment or cost consideration. The control difficulties caused by these uncertainties can be removed using adaptive method along with the backstepping procedure. In this thesis, the adaptive law deals with a constant disturbance $\theta$ in terms of mechanical system load uncertainty. Adaptive law is represented as a differential equation of $\theta$ and is designed using Lyapunov stability method to minimize 
the difference between real and estimated outputs. The estimated disturbance $\hat{\theta}$ will be updated each sample time step and approaches the real value eventually.

In $\mathrm{ABC}$, the whole CLF, which expands step by step, consists of the elements including quadratic terms of the errors between virtual controls, stabilizing function and the unknown parameter errors. The stabilizing functions acting as control laws make the derivatives of CLF negative definite, hence the asymptotic stability of the error system is validated. AOBC which deals with output feedback is more complicated than ABC. For $\mathrm{AOBC}$, an observer is designed to estimate non-measurable states, and the control law consists of those estimated states and the measured output of the system. For the AOBC, besides the elements mentioned above, quadratic observer errors are also included in CLF.

\section{3 Contributions of the thesis}

This thesis applies advanced control methods $\mathrm{ABC}$ and $\mathrm{AOBC}$ to an $\mathrm{AMB}$ system to regulate the position of the AMB's fast-spinning rotor. Detailed and complete procedures of deriving the two strategies are offered. $A B C$ is based on the three states of the linearized model of the AMB: displacement, velocity and current. The adaptive backstepping method will be introduced during the derivation of the $\mathrm{ABC}$. $\mathrm{AOBC}$ is constructed on the assumption that only one position output of the system is measurable. One of the major contributions of this thesis is that the system's global stability has been directly verified by Lyapunov's direct method through the process of control law design. 
In addition, in this thesis, the external disturbance can be estimated by the adaptive laws while other adaptive controllers reported on the AMB system in current literature only estimate uncertain parameters. Due to the adaptive law's on-line estimation of the disturbance, the system shows high disturbance rejection ability. The tuning of the coefficients of controller is discussed in this thesis and the simulations verified the effectiveness of the tuning. But in published papers, the tuning of controller parameters is almost absent. The robustness of the adaptive backstepping controller against parameter uncertainties is verified as well. It should be mentioned that a nonlinear $\mathrm{ABC}$ has been applied in [22]. The nonlinear $\mathrm{ABC}$ treated the coil current as input and all the parameters that are associated with the position of the mass are taken as unknown dynamics. The problem stated in [22] is different from the one in this thesis since our input is the voltage and we only assume an external disturbance as an unknown parameter. In addition, backstepping method has been implemented in [2], where it is combined with ADRC to regulate the rotor displacement through current feedback control. A steady-state error occurred in the simulation result of displacement in [2]. In this thesis, the $\mathrm{ABC}$ is constructed based on position feedback, and there is no steady-state error in the displacement.

\section{4 Thesis outline}

This thesis is organized as follows. In Chapter II, the nonlinear model of the AMB is linearized. State equations are used to construct the linearized model. It is shown in this chapter that the AMB system is inherently unstable. 
In Chapter III, we apply ABC to the unstable AMB system. Both backstepping controller and adaptive laws are developed in this chapter. In order to verify the effectiveness of the controller, we simulate the closed-loop control system on the linearized AMB system. The simulation results demonstrate the effectiveness of controller. At the end of Chapter III, the closed-loop system's robustness is analyzed and a comparison between PID and $\mathrm{ABC}$ for the same $\mathrm{AMB}$ model is conducted.

In Chapter IV, AOBC is applied to the AMB. The equation development for such a control strategy is developed. An exponentially convergent estimator is used to observe the unknown system states. The AOBC is simulated on the linearized model of the AMB. By changing the disturbance value and plant's parameters, the robustness of the closeloop system is tested. At the end of the chapter, we compared the response of AOBC controlled AMB with the one of PID.

Chapter V makes the conclusions and suggests future research directions. 


\section{CHAPTER II}

\section{MODELING OF ACTIVE MAGNETIC BEARING}

\section{1 Principles}

In a typical stable AMB model, the rotor is levitated at its equilibrium point which is positioned right in the middle of two magnets. The two opposite electro magnets are trying to pull the rotor on each side in the absence of any external force. When an external force causes a vibration of the rotor, it will be sensed by the position sensors. Position sensor sends the position information to the electronic control system, which increases the current in one direction and decreases the current in another direction through the respective electro magnets. This produces a force to push the rotor to its original position. The signal from the electronic controller continuously updates the differential force to stabilize the rotor till no position error is sensed. In the following, we 
will discuss the deducing process through physical analyses, and then to model the dynamics of the AMB system.

First, we take a simple magnetic actuator as an example to calculate the forces generated by the current (electromagnetic force).

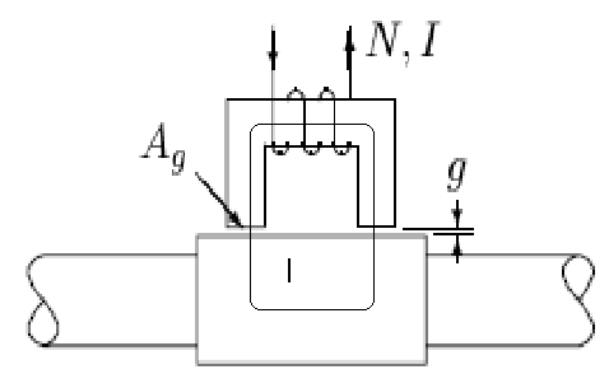

Figure 1: Magnetic actuator [1]

In Figure 1, $I$ is the coil current, $g$ is air gap, $N$ is the number of coil rounds on the core, $A_{g}$ represents the cross-section area and $g$ is the air gap, $l$ is length of the path enclosing a surface through which the current flows. The magnetic field generated by the current will create an upward force. According to Ampere's loop law, we have the following equation, where $H$ is the magnetomotance (magnetic field), which involves the flux density $B, n_{s}$ is the number of the segments through the path $l$ in which $H$ is constant, and $n_{c}$ is the number of different coils that may exist.

$$
\sum_{i=1}^{n_{s}} H_{i} l_{i}=\sum_{i=1}^{n_{c}} N_{i} I_{i}
$$

Assuming that the permeability of the mediums $\mu$ is constant in each segment, we will have the flux density as: 


$$
B_{i}=\mu_{i} H_{i}
$$

Combining (1) and (2) yields

$$
\sum_{i=1}^{n_{s}} \frac{B_{i} l_{i}}{\mu_{i}}=\sum_{i=1}^{n_{c}} N_{i} I_{i}
$$

For the system in Figure 1, because there are two air gaps and the permeability of air $\left(\mu_{g}\right)$ is much less than that of iron $\left(\mu_{0}\right)$, the terms $\frac{B l_{0}}{\mu_{0}}$ can be ignored, where $l_{0}$ means the length of the magnetic flux path in the core. Given (2), we will have

$$
2 \frac{B_{g} g}{\mu_{g}}=N I \Rightarrow B_{g}=\frac{\mu_{g} N I}{2 g}
$$

The energy $E$ stored in the air gaps is represented by

$$
E=\frac{1}{2} B_{g} H_{g} A_{g} 2 g
$$

The electromagnetic force is the derivative of the energy $E$ with respect to air gap. It can be expressed as

$$
f=\frac{d E}{d g}=B_{g} H_{g} A_{g}=\frac{1}{\mu_{g}} B_{g}{ }^{2} A_{g}
$$

With the equation of flux density in (4), we can rewrite (6) as

$$
f=\frac{1}{\mu_{g}} A_{g}\left(\frac{\mu_{g} N I}{2 g}\right)^{2}=\frac{\mu_{g} N^{2} I^{2} A_{g}}{4 g^{2}}
$$




\section{2 Nonlinear model of AMB and its linearization}

In this thesis, we use a one degree of freedom (DOF) AMB model [9] as Figure 2

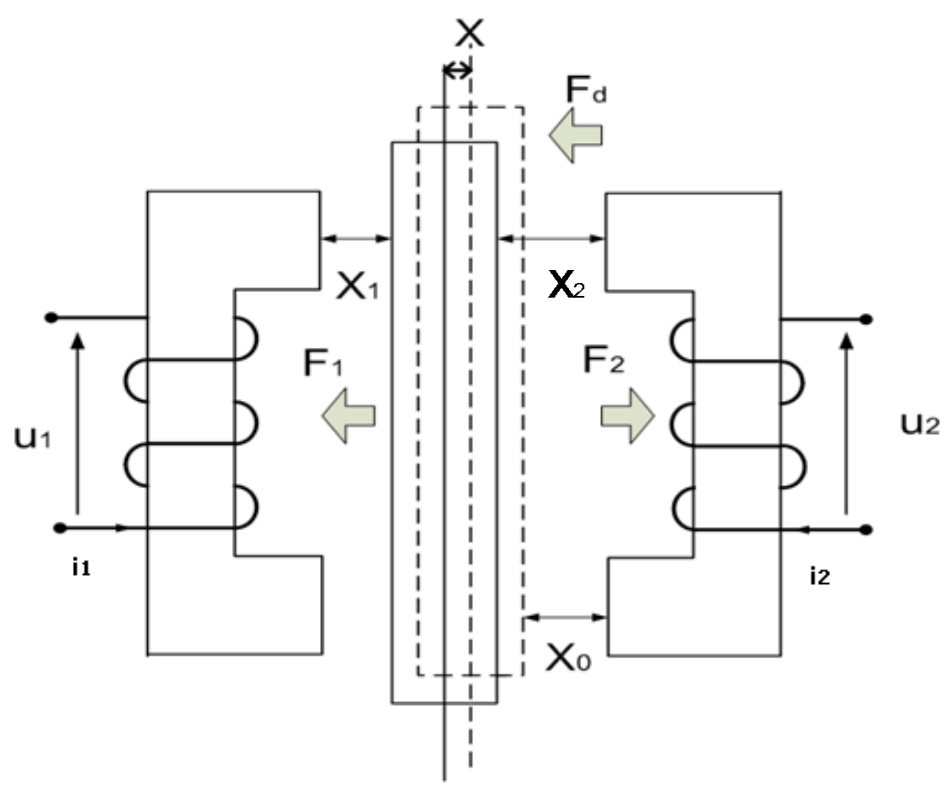

Figure 2: AMB model

There are two opposite forces $F_{1}$ and $F_{2}$. The values of these forces are calculated by (7).

The rotor in the middle of two cores is levitated and rotates in a plane perpendicularly to the figure. We can adjust the input voltage $u_{1}$ and $u_{2}$ to control the two currents $i_{1}$ and $i_{2}$ so as to determine the resultant force. In Figure 2, the displacement of rotor from nominal position $x_{0}$ is $x$, and $m$ is the rotor's mass. In the following part, we will derive the dynamics of the AMB system.

According to Newton's law, we have 


$$
m \ddot{x}=F_{1}+F_{d}-F_{2}
$$

In Figure 2, $x_{1}$ and $x_{2}$ are the air gaps between the rotor and left and right stators respectively. Replacing $g$ in (7) with $x_{1}$ and $x_{2}$ separately, we can derive the two electromagnetic forces $F_{1}$ and $F_{2}$ as follows

$$
F_{1}=\frac{\mu_{g} N^{2} i_{1}^{2} A_{g}}{4 x_{1}^{2}}=\frac{K}{4}\left(\frac{i_{1}}{x_{1}}\right)^{2} \quad F_{2}=\frac{\mu_{g} N^{2} i_{2}^{2} A_{g}}{4 x_{2}^{2}}=\frac{K}{4}\left(\frac{i_{2}}{x_{2}}\right)^{2}
$$

where $K=\mu_{g} N^{2} A_{g}$. According to Kirchoff's Voltage Law (KVL), we have

$$
u_{1}=R i_{1}+L_{s} \frac{d i_{1}}{d t}+\frac{K}{2} \frac{d}{d t}\left(\frac{i_{1}}{x_{1}}\right) \quad u_{2}=R i_{2}+L_{s} \frac{d i_{2}}{d t}+\frac{K}{2} \frac{d}{d t}\left(\frac{i_{2}}{x_{2}}\right)
$$

In (10), the first term on the right represents the voltage that is produced by coil resistance $R$. The second term is because of the coil self inductance $L_{s}$. The third term models the back electromotive force (back EMF) generated by the air gap flux change.

We suppose $\left(x_{0}, i_{0}, u_{0}\right)$ represents nominal states. $x_{1}, i_{1}$ and $u_{1}$ are defined as position, current and voltage of the AMB in one side. Then we will have

$$
\begin{array}{ll}
x_{1}=x_{0}-x & x_{2}=x_{0}+x \\
i_{1}=i_{0}+i & i_{2}=i_{0}-i \\
u_{1}=u_{0}+u & u_{2}=u_{0}-u
\end{array}
$$

Substituting (11)-(13) into (10) and substituting (9) into (8), we will have a nonlinear system model given by 


$$
\begin{aligned}
& \dot{x}=v \\
& \dot{v}=\frac{K}{4 m}\left(\frac{i_{1}}{x_{0}-x}\right)^{2}-\frac{K}{4 m}\left(\frac{i_{2}}{x_{0}+x}\right)^{2}+\frac{F_{d}}{m} \\
& \dot{i_{1}}=\frac{2\left(x_{0}-x\right)}{2 L_{s}\left(x_{0}-x\right)+K}\left[-R i_{1}-\frac{K}{2\left(x_{0}-x\right)^{2}} v i_{0}+u_{1}\right] \\
& \dot{i}_{2}=\frac{2\left(x_{0}+x\right)}{2 L_{s}\left(x_{0}+x\right)+K}\left[-R i_{2}-\frac{K}{2\left(x_{0}+x\right)^{2}} v i_{1}+u_{2}\right]
\end{aligned}
$$

We use Jacobian transformation to linearize the nonlinear model (14) around the equilibrium state. The details of the linearization can be found in [2]. The linearized state equations of system configuration used to generate controller is given as follows. We represent the state matrix as $A$. Electromagnets are biased with a current $i_{0}$. According to [9], as the current $i_{0}$ is a constant, the bias voltage due to the coil resistance $R$ is $u_{0}=R i_{0}$. As the current $i_{0}$ is varying, the relationship between the $i_{0}$ and $u_{0}$ is represented by (16) [9].

$$
\begin{aligned}
{\left[\begin{array}{c}
\dot{x} \\
\dot{v} \\
\dot{i}
\end{array}\right]=} & \underbrace{\left[\begin{array}{ccc}
0 & 1 & 0 \\
\frac{2 k_{s}}{m} & 0 & \frac{2 k_{i}}{m} \\
0 & \frac{-k_{i}}{L_{0}+L_{s}} & \frac{-R}{L_{0}+L_{s}}
\end{array}\right]}_{A}\left[\begin{array}{c}
x \\
v \\
i
\end{array}\right]+\left[\begin{array}{c}
0 \\
0 \\
\frac{1}{L_{0}+L_{s}}
\end{array}\right] u+\left[\begin{array}{c}
0 \\
\frac{1}{m} \\
0
\end{array}\right] F_{d} \\
& \frac{d}{d t} i_{0}=\frac{-R}{L_{0}+L_{s}} i_{0}+\frac{1}{L_{0}+L_{s}} u_{0}
\end{aligned}
$$

where $k_{s}=\frac{K}{2} \frac{i_{0}^{2}}{x_{0}^{3}}, k_{i}=\frac{K}{2} \frac{i_{0}}{x_{0}^{2}}$ and $L_{0}=\frac{K}{2 x_{0}}$. Equation (16) depicts a bias current dynamic model. From the linear system (15), we can calculate the eigenvalues of $A$ : 
[207.5781 -179.4896-70.1937]. Since there is a positive eigenvalue for matrix $A$, we can claim that the system is inherently unstable. An effective controller is crucial to stabilize the AMB. We consider that the initial conditions are zero.

After the linearization and the decoupling steps which separate nominal states $x_{0}, i_{0}, u_{0}$ from $x, i, u$, we can design controller based on (16). The block diagram representation of the AMB system expressed by (15) and (16) is given in Figure 3.

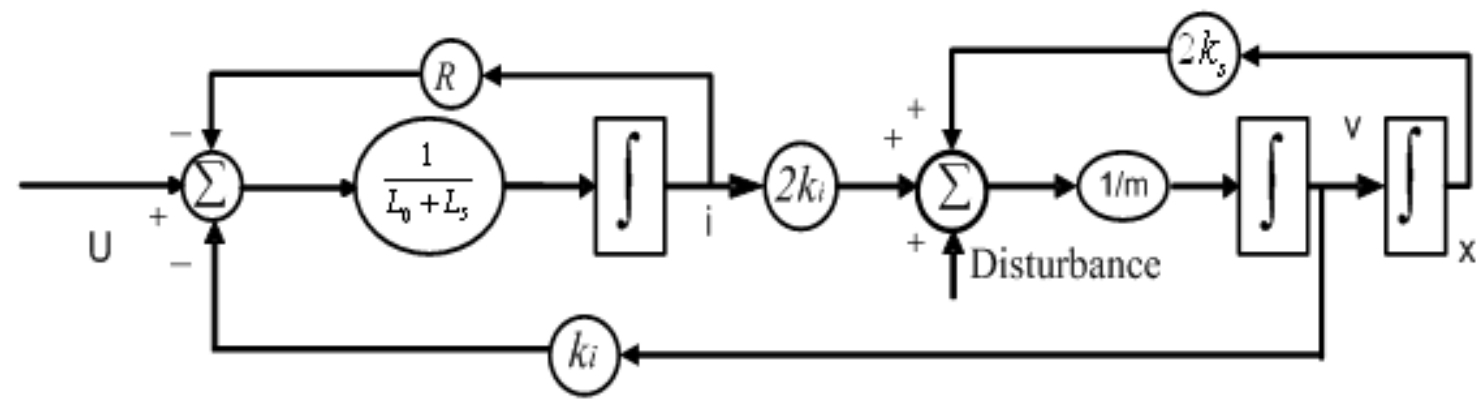

Figure 3: Block diagram of open-loop AMB plant

In Figure 3, system's dynamic is described by (15). The voltage control input $u$, which is directly controls the current, has three integrations between position displacement. The left side of the block diagram is electrical subsystem which depicts how input influences the current through inductance. The right side of the block diagram is electro mechanical subsystem, where the position displacement is governed directly by the current. An external disturbance signal, functioning as a step input, is added to the mechanical subsystem. The configurations of the system parameters are listed in Table 1. 
Table 1: Parameter configurations

\begin{tabular}{|c|c|c|}
\hline Parameter & Symbol & Value(unit) \\
\hline $\begin{array}{c}\text { Force-Displacement } \\
\text { Constant }\end{array}$ & $K_{s}$ & $142860 \mathrm{~N} / \mathrm{m}$ \\
\hline $\begin{array}{c}\text { Force-Current } \\
\text { Constant }\end{array}$ & $K_{i}$ & $100 \mathrm{~N} / \mathrm{A}$ \\
\hline Coil Self Inductance & $L_{s}$ & $120 \mathrm{mH}$ \\
\hline Air Gap Inductance & $L_{0}$ & $70 \mathrm{mH}$ \\
\hline Weight of Rotor & $m$ & $4.6 \mathrm{~kg}$ \\
\hline Coil Resistance & $R$ & 8 \\
\hline Nominal Air Gap & $x_{0}$ & $0.0007 \mathrm{~m}$ \\
\hline Bias current & $i_{0}$ & $1 \mathrm{~A}$ \\
\hline Disturbance Force & $F_{d}$ & $4.6 \mathrm{~N}$ \\
\hline
\end{tabular}

\section{3 Summary of the chapter}

This chapter used basic physical principles to construct a one DOF AMB model. The development of the linearized state equations for the AMB is given. Because the linearized model is unstable, the AMB system has to be stabilized. We will employ ABC and $\mathrm{AOBC}$ to control and stabilize the $\mathrm{AMB}$ respectively. The controller design will be explained in the next two chapters. 


\section{CHAPTER III}

\section{ADAPTIVE BACKSTEPPING CONTROL WITH FULL-STATE FEEDBACK}

In this chapter, the $\mathrm{ABC}$ is developed in details. Then it is applied to and simulated on the AMB model. An investigation of the control system's robustness is discussed. The tuning of the parameters of the controller is also introduced in this chapter.

\section{1 Control objective and strategy}

Since AMB is an unstable system, the primary control objectives are to stabilize the AMB and to drive the position of the rotor to its equilibrium point in the presence of an external force with the knowledge of three states feedback. In addition, the control system must be robust against external disturbance and the uncertainties of the physical system. It is also 
desired that disturbance be estimated accurately so it can be canceled in the control effort.

Adaptive backstepping controller consists of two parts: backstepping controller and adaptive laws. The backstepping controller is used to stabilize and control rotor's position and adaptive law estimates the disturbance. The general control Lyapunov function (CLF) is constructed to include the position displacement, errors between virtual estimates and stabilized functions, and of the difference between estimated and real disturbance. In the design of $\mathrm{ABC}$, it is assumed that the system is in a basic form as following equations which is called "strict feedback form" [24].

$$
\begin{aligned}
& \dot{x}=f(x)+g(x) \xi_{1} \\
& \dot{\xi}_{1}=f_{1}\left(x, \xi_{1}\right)+g_{1}\left(x, \xi_{1}\right) \xi_{2} \\
& \dot{\xi}_{2}=f_{2}\left(x, \xi_{1}, \xi_{2}\right)+g_{2}\left(x, \xi_{1}, \xi_{2}\right) \xi_{3} \\
& \quad \vdots \\
& \dot{\xi}_{k-1}=f_{k-1}\left(x, \xi_{1} \cdots \xi_{k-1}\right)+g_{k-1}\left(x, \xi_{1} \cdots \xi_{k-1}\right) \xi_{k} \\
& \dot{\xi}_{k}=f_{k}\left(x, \xi_{1} \cdots \xi_{k}\right)+g_{k}\left(x, \xi_{1} \cdots \xi_{k}\right) u
\end{aligned}
$$

Equation (17) is a general system in strict feedback form, in which $x \in R^{n}$ and the rest of the states $\xi_{i}(i=1 \cdots k)$ are scalars, functions $f$ and $g$ only depend on the previous state variables that are fed back to the current states. When we design the $A B C$, the state equations of AMB system should be transformed to the form like (17).

Four components constitute a typical closed-loop ABC system as Figure 4. In the figure, position, velocity and current sensors are used to measure the system's three states and transfer them to the $\mathrm{ABC}$. The amplifier acts as a proportional part of control signal which usually occurs in some practical situation, however, it is not used in the thesis. 


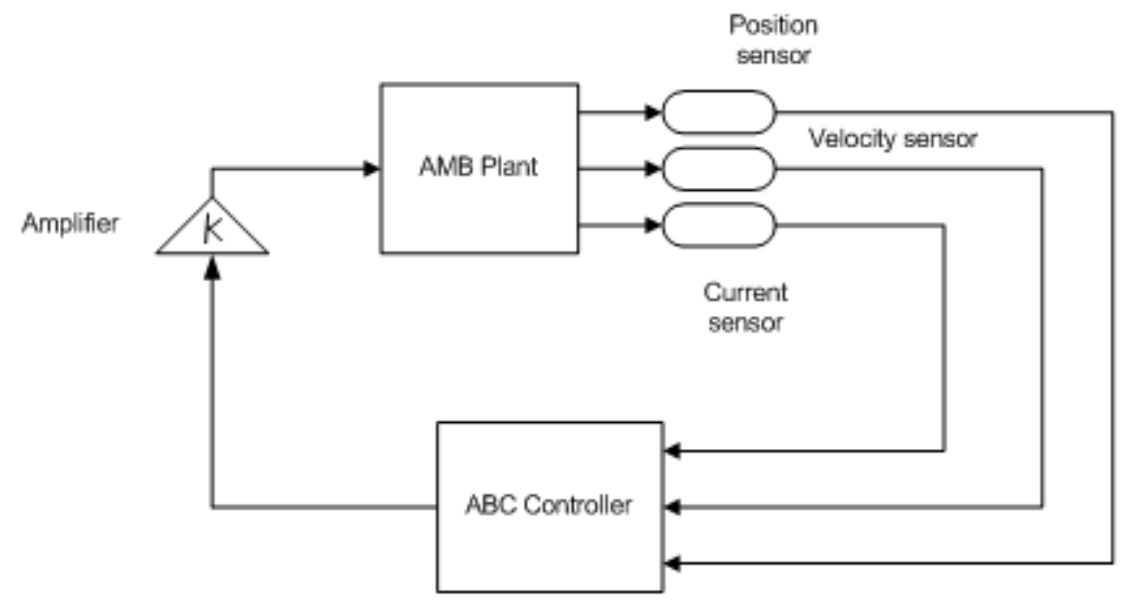

Figure 4: Model of $A B C$ of $A M B$

\section{2 Model transformation}

The state equations of AMB (15) can be represented as

$$
\left[\begin{array}{c}
\dot{x} \\
\dot{v} \\
\dot{i}
\end{array}\right]=\left[\begin{array}{lll}
0 & 1 & 0 \\
a & 0 & b \\
0 & c & d
\end{array}\right]\left[\begin{array}{l}
x \\
v \\
i
\end{array}\right]+\left[\begin{array}{l}
0 \\
0 \\
e
\end{array}\right] u+\left[\begin{array}{l}
0 \\
f \\
0
\end{array}\right] F_{s}
$$

where $a=\frac{2 k_{s}}{m}, b=\frac{2 k_{i}}{m}, c=\frac{-k_{i}}{L_{0}+L_{s}}, d=\frac{-R}{L_{0}+L_{s}}, e=\frac{1}{L_{0}+L_{s}}, f=\frac{1}{m}$. For creating a "strict feedback form", equation (18) can be transformed into (19), (20) and (21), where the $x_{1}=\frac{1}{b} x, x_{2}=\frac{1}{b} v, x_{3}=i$.

$$
\begin{aligned}
& \dot{x}_{1}=x_{2} \\
& \dot{x}_{2}=x_{3}+\frac{a}{b} x_{1}+\theta=x_{3}+\varphi_{1}\left(\theta, x_{1}\right) \\
& \dot{x}_{3}=u^{\prime}+c b x_{2}+d x_{3}=u^{\prime}+\varphi_{2}
\end{aligned}
$$


In (19), (20) and (21), the control and the disturbance force are defined as $\theta=\frac{F_{s}}{b m}=0.023, u^{\prime}=\frac{1}{e} u$

\section{3 Design procedure}

In this section, adaptive laws and backstepping design are introduced separately. Our control goal is to regulate the position of the rotor $x_{1}$. For (19), we suppose that the virtual control $x_{2}$ can be used to drive $x_{1}$ to zero. Then we take $\alpha_{1}=-c_{1} x_{1}$ as stabilizing function or virtual controller (virtual state) to replace $x_{2}, c_{i}(i=1,2,3)$ are positive numbers. If $x_{2}=\alpha_{1}$, the desired state $x_{1}$ will be asymptotically stable by constructing the $\mathrm{CLF}_{V}=\frac{1}{2} x_{1}^{2}\left(\dot{V}=-c_{1} x_{1}^{2}\right)$. Since there must be an error between $x_{2}$ and $\alpha_{1}$, we need to construct new state space equations called "error system" [13] whose states are the differences between the real states and stabilizing functions, and drive the error states to zeros. The control goal then becomes asymptotically stabilizing all the states of the error system. In the AMB system, rotor displacement $x_{1}$ needs to be driven to zero. We consider the displacement $x_{1}$ as the first state $z_{1}$ of the error system, hence $z_{1}=x_{1}$, and the error between second state $x_{2}$ and its stabilizing function $\alpha_{1}$ as $z_{2}=x_{2}-\alpha_{1}$. Then the CLF consisting of these two values is

$$
V_{1}=\frac{1}{2} z_{1}^{2}+\frac{1}{2} z_{2}^{2}
$$

Since (22) is the CLF for (19) and (20), our task is to find a suitable input denoted by the virtual control $x_{3}$ to make the derivative of (22) negative definite so that the two 
terms $z_{1}$ and $z_{2}$ will be driven to zero eventually. Even if it is negative semi-definite, LaSalle-Yoshizawa theorem [13] shows that $x_{1}$ will still be driven zero.

With (19) and (20), the derivative of (22) is

$$
\dot{V}_{1}=x_{1} x_{2}+z_{2}\left(x_{3}+\varphi_{1}-\frac{\partial \alpha_{1}}{\partial x_{1}} x_{2}\right)
$$

We choose $x_{3}$ as virtual control signal. If the second stabilization function is given by

$$
\alpha_{2}=x_{3}=-c_{2} z_{2}-\varphi_{1}+\frac{\partial \alpha_{1}}{\partial x_{1}} x_{2}-z_{1}
$$

the derivative of $V_{1}$ will become $-c_{1} z_{1}^{2}-c_{2} z_{2}^{2}$ which is negative semi-definite. However, there is still an error $z_{3}=x_{3}-\alpha_{2}$. So a new CLF including all the existing errors and displacement is created as

$$
V_{2}=\frac{1}{2} z_{1}^{2}+\frac{1}{2} z_{2}^{2}+\frac{1}{2} z_{3}^{2}
$$

The derivative of $V_{2}$ is

$$
\begin{aligned}
\dot{V}_{2} & =-c_{1} z_{1}^{2}-c_{2} z_{2}^{2}+z_{2} z_{3}+z_{3}\left(u+\varphi_{2}-\dot{\alpha}_{2}\right) \\
& =-c_{1} z_{1}^{2}-c_{2} z_{2}^{2}+z_{3}\left(z_{2}+u^{\prime}+\varphi_{2}-\dot{\alpha}_{2}\right)
\end{aligned}
$$

where $u^{\prime}$ is chosen as

$$
u^{\prime}=-c_{3} z_{3}-z_{2}-\varphi_{2}+\dot{\alpha}_{2}
$$


Then the derivative of the CLF (26) of the system can be rewritten as

$$
\dot{V}_{2}=-c_{1} z_{1}^{2}-c_{2} z_{2}^{2}-c_{3} z_{3}^{2}
$$

which means the derivative of the final CLF is negative semi definite. So the control goal is achieved.

The above procedure is under the consumption that no external disturbance exists. If there is one, we will have to generate an adaptive law to make estimate error of disturbance to be zero so as to obtain an accurate value of the disturbance and compensate it. The estimated disturbance will be additional feedback information in control law. The details about disturbance estimation are given as follows.

Let disturbance be $\theta$, and estimated disturbance be $\hat{\theta}_{1}$. We will have an estimation error $\tilde{\theta}_{1}=\theta-\hat{\theta}_{1}$. We add the quadratic form of it to (23) and then form a new CLF (29). Positive numbers $\gamma_{i}(i=1,2,3)$ are chosen as adaptive coefficients.

$$
V_{1}=\frac{1}{2} z_{1}^{2}+\frac{1}{2} z_{2}^{2}+\frac{1}{2 \gamma_{1}} \hat{\theta}_{1}^{2}
$$

Because of the disturbance, the second part in (23) becomes $z_{2}\left(x_{3}+\varphi_{1}-\frac{\partial \alpha_{1}}{\partial x_{1}} x_{2}+\theta\right)$. We reselect $\alpha_{2}$ as

$$
\begin{aligned}
\alpha_{2} & =-z_{1}-c_{2} z_{2}-\varphi_{1}\left(x_{1}\right)-\hat{\theta}_{1}+\dot{\alpha}_{1} \\
& =-\left(c_{1} c_{2}+\varphi_{1}+1\right) x_{1}-\left(c_{1}+c_{2}\right) x_{2}-\hat{\theta}_{1}
\end{aligned}
$$


Then the derivative of the CLF becomes

$$
\begin{aligned}
\dot{V}_{1} & =-c_{1} z_{1}^{2}-c_{2} z_{2}^{2}+z_{2} \tilde{\theta}_{1}+z_{3} z_{2}-\frac{1}{\gamma_{1}} \dot{\hat{\theta}}_{1} \tilde{\theta}_{1} \\
& =-c_{1} z_{1}^{2}-c_{2} z_{2}^{2}+z_{3} z_{2}+\tilde{\theta}_{1}\left(z_{2}-\frac{1}{\gamma_{1}} \dot{\hat{\theta}}_{1}\right)
\end{aligned}
$$

In (31), if we choose adaptive law as $\dot{\hat{\theta}}_{1}=\gamma_{1} z_{2}$, it will make the derivative function of $V_{1}$ negative definite assuming the $z_{2} z_{3}$ term could be cancelled in the future. Next we need to calculate the derivative of $V_{2}$ that includes $\alpha_{2}$. Considering external disturbance, the derivative of $\alpha_{2}$ becomes

$$
\begin{aligned}
\dot{\alpha}_{2} & =\frac{\partial \alpha_{2}}{\partial z_{1}} \dot{z}_{1}+\frac{\partial \alpha_{2}}{\partial x_{2}} \dot{x}_{2}+\frac{\partial \alpha_{2}}{\partial \hat{\theta}_{1}} \dot{\hat{\theta}}_{1} \\
& =\frac{\partial \alpha_{2}}{\partial z_{1}} \dot{z}_{1}+\frac{\partial \alpha_{2}}{\partial x_{2}}\left(x_{3}+\varphi_{1}\left(x_{1}\right)+\theta\right)+\frac{\partial \alpha_{2}}{\partial \hat{\theta}_{1}} \dot{\hat{\theta}}_{1}
\end{aligned}
$$

In (32), there is still a disturbance $\theta$ existing in $\dot{\alpha}_{2}$ which can not be replaced by $\hat{\theta}_{1}$.

Employ $\hat{\theta}_{2}$ instead of $\theta$. Therefore, the whole CLF can be reconstructed as

$$
V_{2}=\frac{1}{2} z_{1}^{2}+\frac{1}{2} z_{2}^{2}+\frac{1}{2} z_{3}^{2}+\frac{1}{2 \gamma_{1}} \tilde{\theta}_{1}^{2}+\frac{1}{2 \gamma_{2}} \tilde{\theta}_{2}^{2}
$$

The control law that was derived before is repeated as follows.

$$
u^{\prime}=-c_{3} z_{3}-z_{2}-\varphi_{2}+\dot{\alpha}_{2}
$$

Substituting (32) into (34) yields 


$$
u^{\prime}=-\left(c_{3}+d\right) x_{3}-\left[c_{3}\left(c_{1} c_{2}+\frac{a}{b}+1\right)+c_{1}\right] x_{1}-\left[c_{3}\left(c_{1}+c_{2}\right)+1+c b\right] x_{2}-c_{3} \hat{\theta}_{1}+\dot{\alpha}_{2}
$$

Given the control law above, the derivative of $V_{2}$ becomes

$$
\dot{V}_{2}=-c_{1} z_{1}^{2}-c_{2} z_{2}^{2}-c_{3} z_{3}^{2}-\frac{1}{\gamma_{2}} \tilde{\theta}_{2} \dot{\hat{\theta}}_{2}-z_{3} \frac{\partial \alpha_{2}}{\partial x_{2}} \tilde{\theta}_{2}
$$

In order to make (36) negative semi definite, we need to eliminate the error parts which contain $\tilde{\theta}_{2}$. If we choose adaptive law as

$$
\dot{\hat{\theta}}_{2}=-z_{3} \gamma_{2} \frac{\partial \alpha_{2}}{\partial x_{2}}
$$

the last two terms of (36) will be eliminated by substituting (37) into (36). Then the derivative of $V_{2}$ becomes

$$
\dot{V}_{2}=-c_{1} x_{1}^{2}-c_{2} z_{2}^{2}-c_{3} z_{3}^{2}
$$

Now that the derivative of the final CLF is negative semi definite, the system will be stabilized at its equilibrium point. The final adaptive backstepping control law is generated by calculating (35) as

$$
\begin{aligned}
u^{\prime}= & -\left(\frac{a}{b}\left(c_{1}+c_{2}+c_{3}\right)+2 c_{1}+c_{3}+c_{1} c_{2} c_{3}\right) x_{1} \\
& -\left(c_{1} c_{2}+c_{2} c_{3}+c_{1} c_{3}+c b+\varphi_{1}+c_{1}+c_{2}+3\right) x_{2} \\
& -\left(c_{1}+c_{2}+c_{3}+d\right) x_{3}
\end{aligned}
$$

The above equation is the final controller which includes three-state feedback.

\section{4 Simulation results}


Since $z_{i}(i=1,2,3)$ represent the states of the error system, we can write the error system's state equation as follows.

$$
\begin{aligned}
& \frac{d}{d t}\left[\begin{array}{l}
z_{1} \\
z_{2} \\
z_{3}
\end{array}\right]=\left[\begin{array}{ccc}
-c_{1} & 1 & 0 \\
-1 & -c_{2} & 1 \\
0 & -1 & -c_{3}
\end{array}\right]\left[\begin{array}{l}
z_{1} \\
z_{2} \\
z_{3}
\end{array}\right]+\left[\begin{array}{cc}
0 & 0 \\
1 & 0 \\
0 & \frac{\partial \alpha_{2}}{\partial x_{2}}
\end{array}\right]\left[\begin{array}{c}
\tilde{\theta}_{1} \\
\tilde{\theta}_{2}
\end{array}\right] \\
& \frac{d}{d t}\left[\begin{array}{l}
\theta_{1} \\
\theta_{2}
\end{array}\right]=\left[\begin{array}{ccc}
0 & \gamma_{1} & 0 \\
0 & 0 & -\gamma_{2} \frac{\partial \alpha_{2}}{\partial x_{2}}
\end{array}\right]\left[\begin{array}{c}
z_{1} \\
z_{2} \\
z_{3}
\end{array}\right]
\end{aligned}
$$

Based on (40), we can draw the diagram of closed-loop adaptive system as Figure 5.

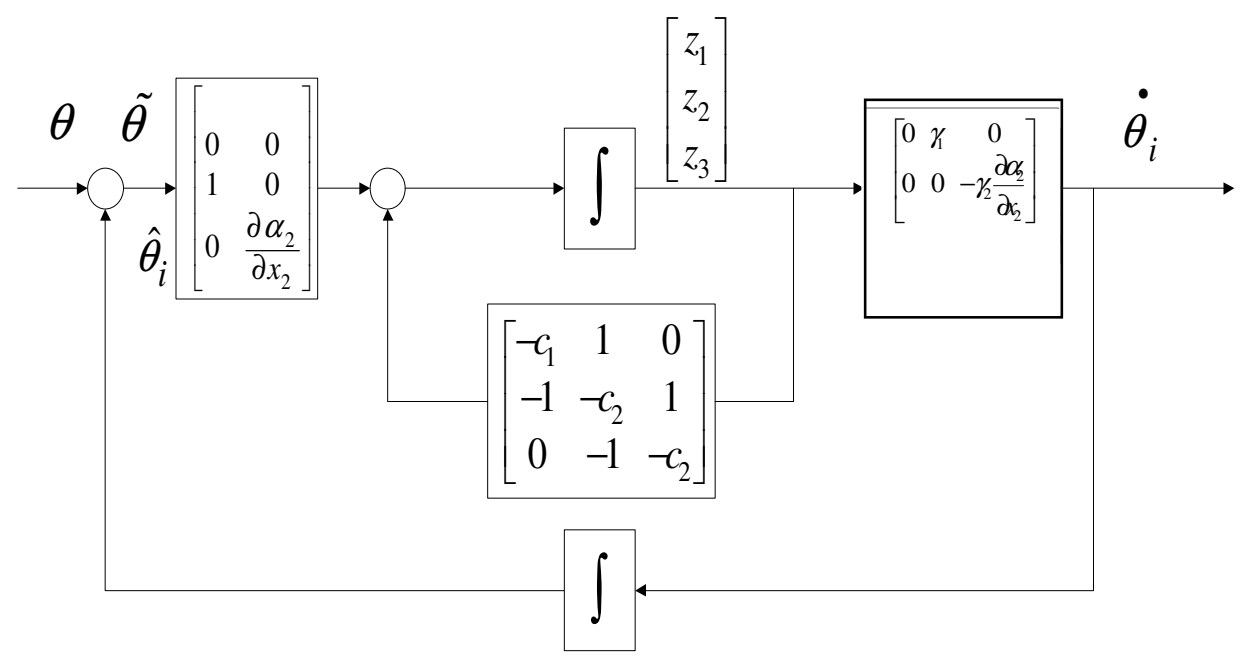

Figure 5: The closed-loop adaptive system

Figure 5 represents the properties of the system after applying control law to it. The constant system matrix has negative diagonal elements, while its off-diagonal elements are skew-symmetric. Each step of the design generates an error variable, a stabilizing function $\alpha_{i}$ and a new estimate $\hat{\theta}_{i}$. 
Using the system parameters shown in Table 1, we can construct the Simulink model for this $\mathrm{ABC}$ controlled $\mathrm{AMB}$ system. We choose simulation time as 5 seconds. The disturbance as a step input is added to the system at $1 \mathrm{~s}$. All the initial values are zero. Including the control law (39), the closed-loop ABC system is illustrated as Figure 6.

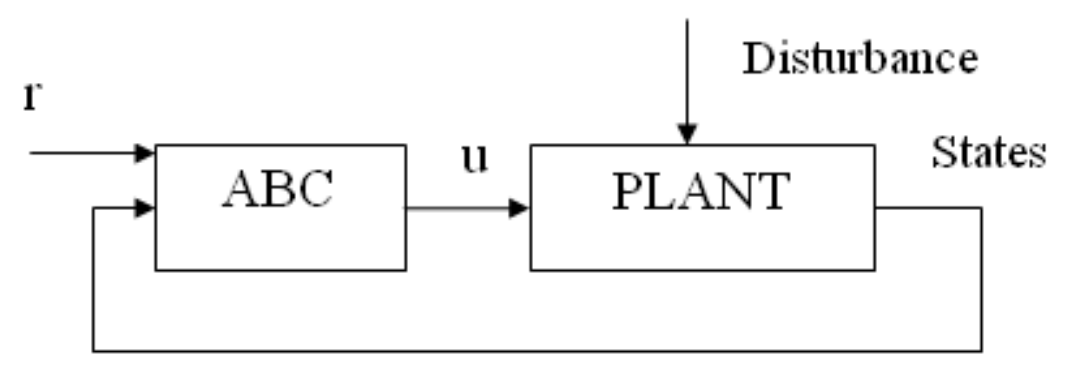

Figure 6: SIMULINK model of ABC

The block "plant" is the unstable AMB model, which has two inputs: control signal $u$ and a disturbance, and three state variables $x, v, i$. The control law is computed in $\mathrm{ABC}$ block to provide input signal of this plant.

In the following part, two sets of simulation results are given with different Lyapunov backstepping coefficients $c_{i}(i=1,2,3)$ (LCs) and adaptive coefficients $\gamma_{i}(i=1,2)$ (ACs) respectively for the purpose of investigating how these coefficients affect the control results of three states, disturbance estimate and control effort. Note that the desired estimation value of disturbance is $\theta=\frac{F_{s}}{b m}=0.023$.

The first set of simulation results is produced and shown in Figures 7, 8, 9, and 10 for which AC values are: $\gamma_{1}=1, \quad \gamma_{2}=1$. The figures shows the time domain response of the 
three states, disturbance estimation and control effort for different LCs We choose LCs values as $c_{1}=c_{2}=c_{3}=500$ and $c_{1}=3000, c_{2}=1000, c_{3}=500$ respectively.
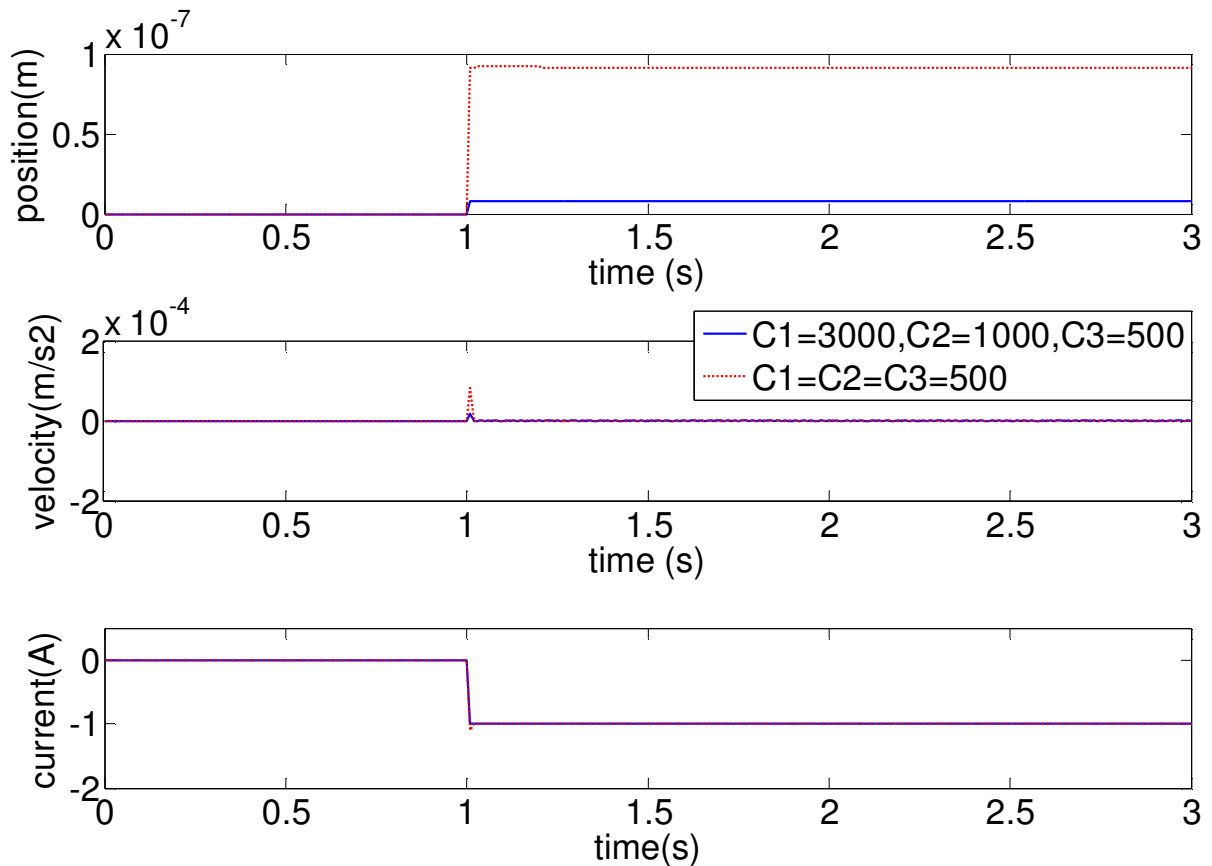

Figure 7: Control results of the three states with different $L C$ 


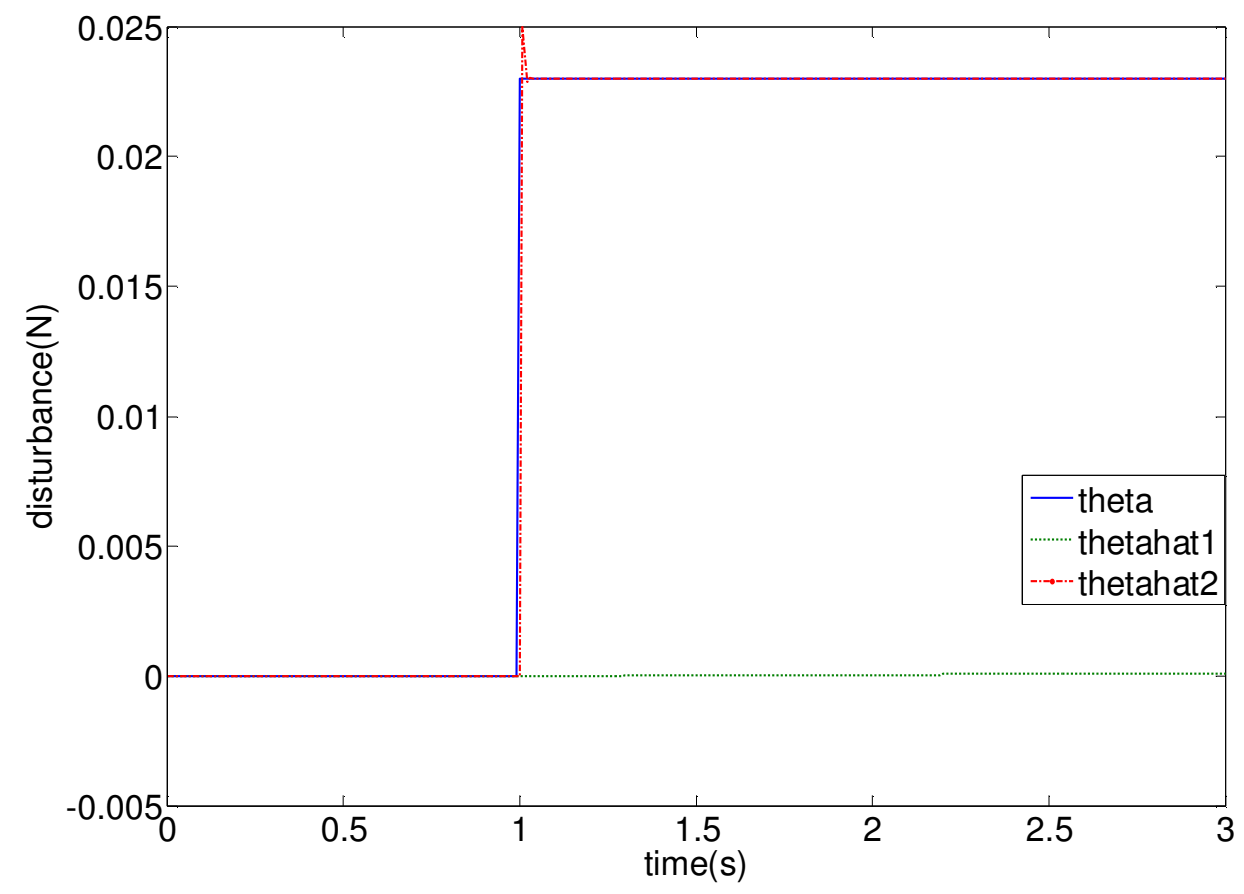

Figure 8: Disturbance $(\theta)$ estimates when $c_{1}=c_{2}=c_{3}=500$

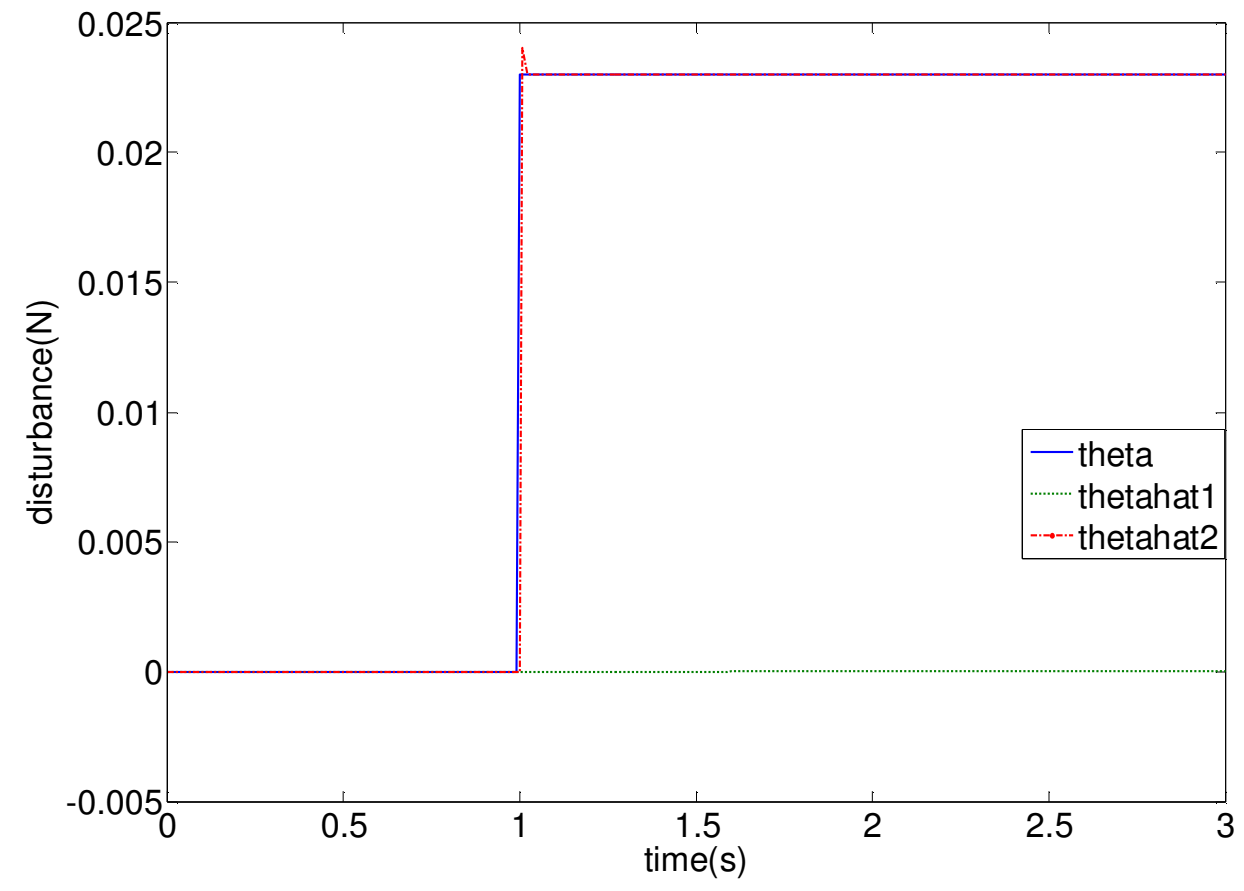

Figure 9: Disturbance and estimates when $c_{1}=3000, c_{2}=1000, c_{3}=500$ 


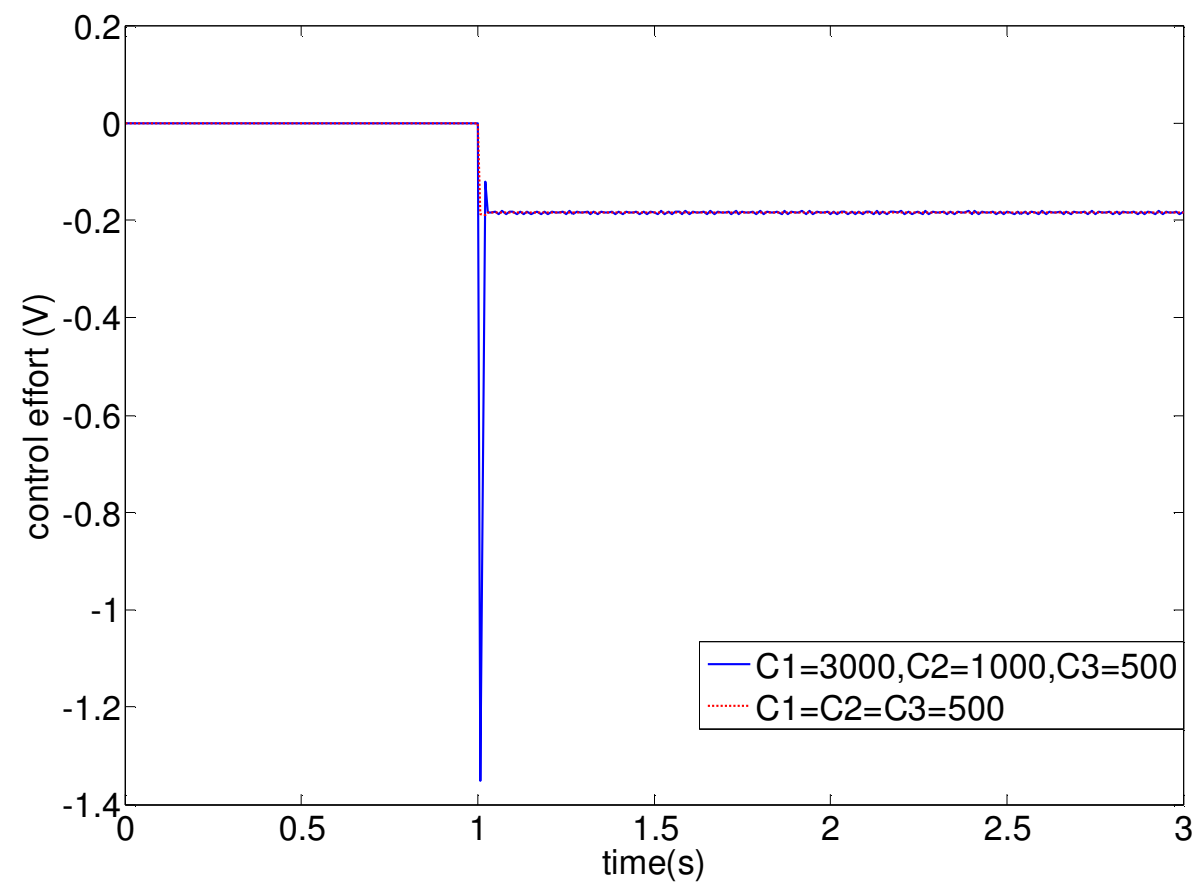

Figure 10: Control effort (voltage) with different LC

From Figure 7 through Figure 10, it is observed that the rotor's displacement is not driven to zero though the fact that the spike value of $x_{1}$ is not exceeding $0.7 \mathrm{~mm}$ that can still guarantee the rotor not touching stator. If we set simulation time long enough, the displacement will converge to zero eventually, and estimators also reach the disturbance value. We can find out that by setting $\mathrm{LC}$ values large without changing $\mathrm{AC}$, the overshoot of the displacement could be remarkably reduced.

In Figure 10, it is noted that the control effort changes rapidly at the time instant when the disturbance jumps from zero to 0.023 (with simulation unit). Such a fast-changing control effort will be very difficult to implement in the real world. However, in reality, the disturbance generally increases gradually from zero to a specific value [22]. 
Therefore, the real rate of change for the control effort will not be as fast as the one shown Figure 10. The control effort for a gradually increased disturbance (ramp disturbance) is given in Figure 11, where the control effort for ramp disturbance changes much slower than the one for step disturbance.

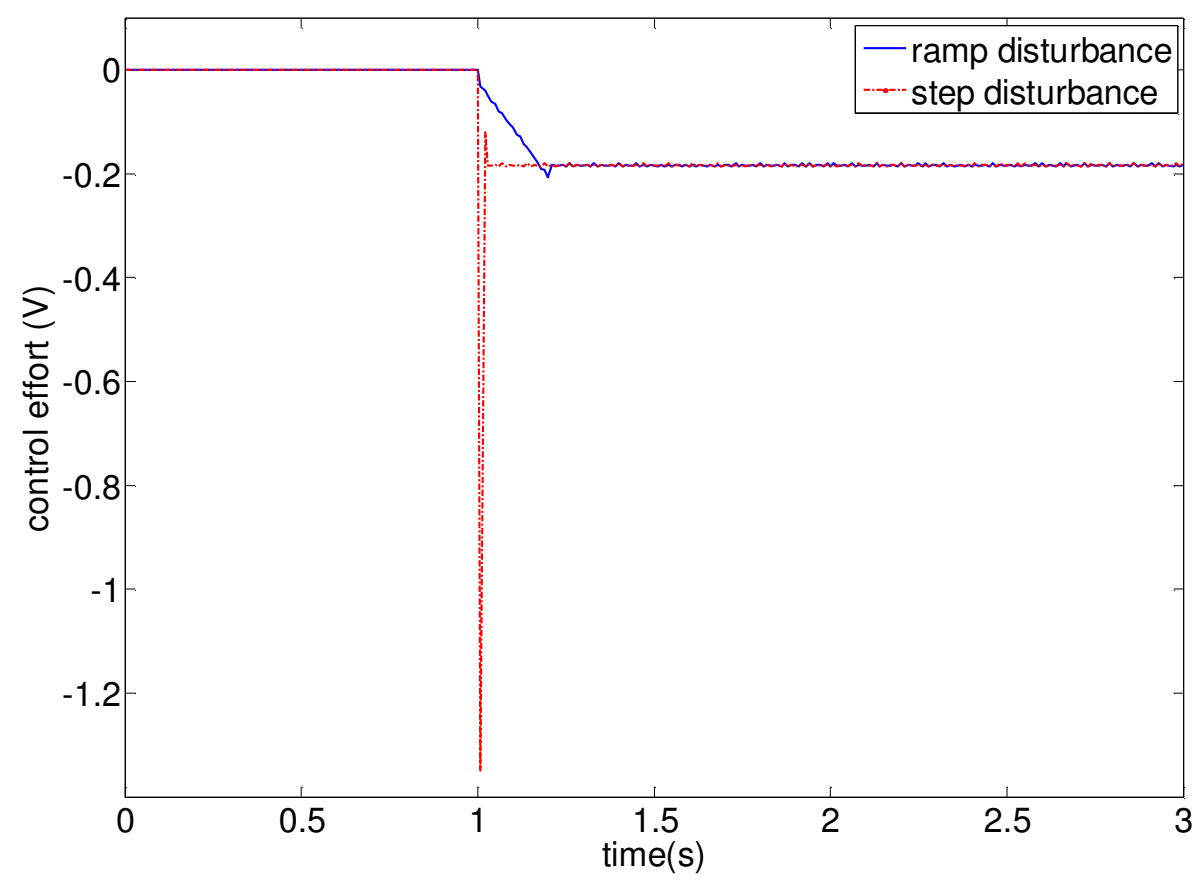

Figure 11: Control efforts with step disturbance and ramp disturbance when

$$
c_{1}=3000, c_{2}=1000, c_{3}=500, \gamma_{1}=1, \gamma_{2}=1
$$

In order to investigate the effect of $\mathrm{AC}$, we change their values in the second set of simulation while leaving LC values unchanged. We choose $c_{1}=3000, c_{2}=1000, c_{3}=500$. We increase the first AC $\gamma_{1}$ from 1 to 3000, and decrease the second AC $\gamma_{2}$ from 1 to 0.1 . The simulation results for different ACs are shown in figures 12 through 15 . 

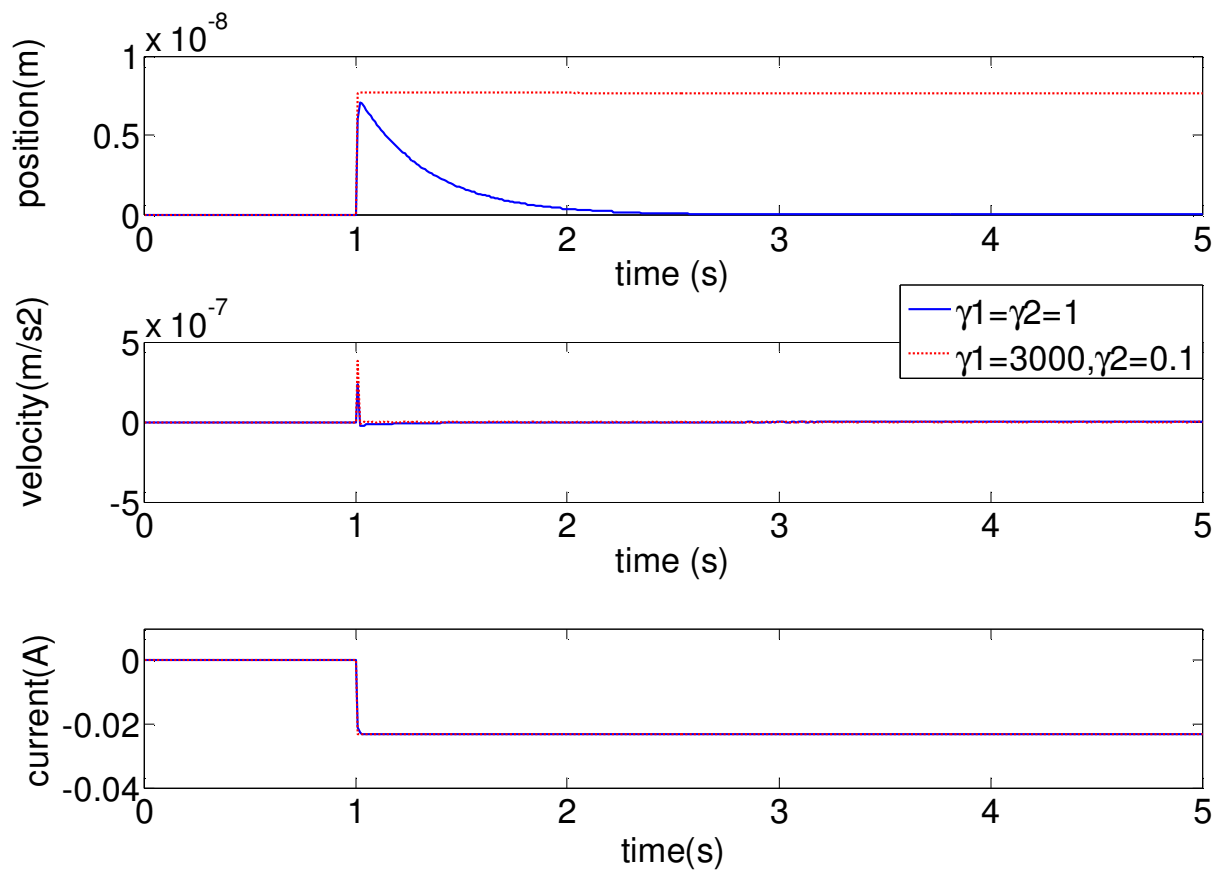

Figure 12: Control result with different $A C$

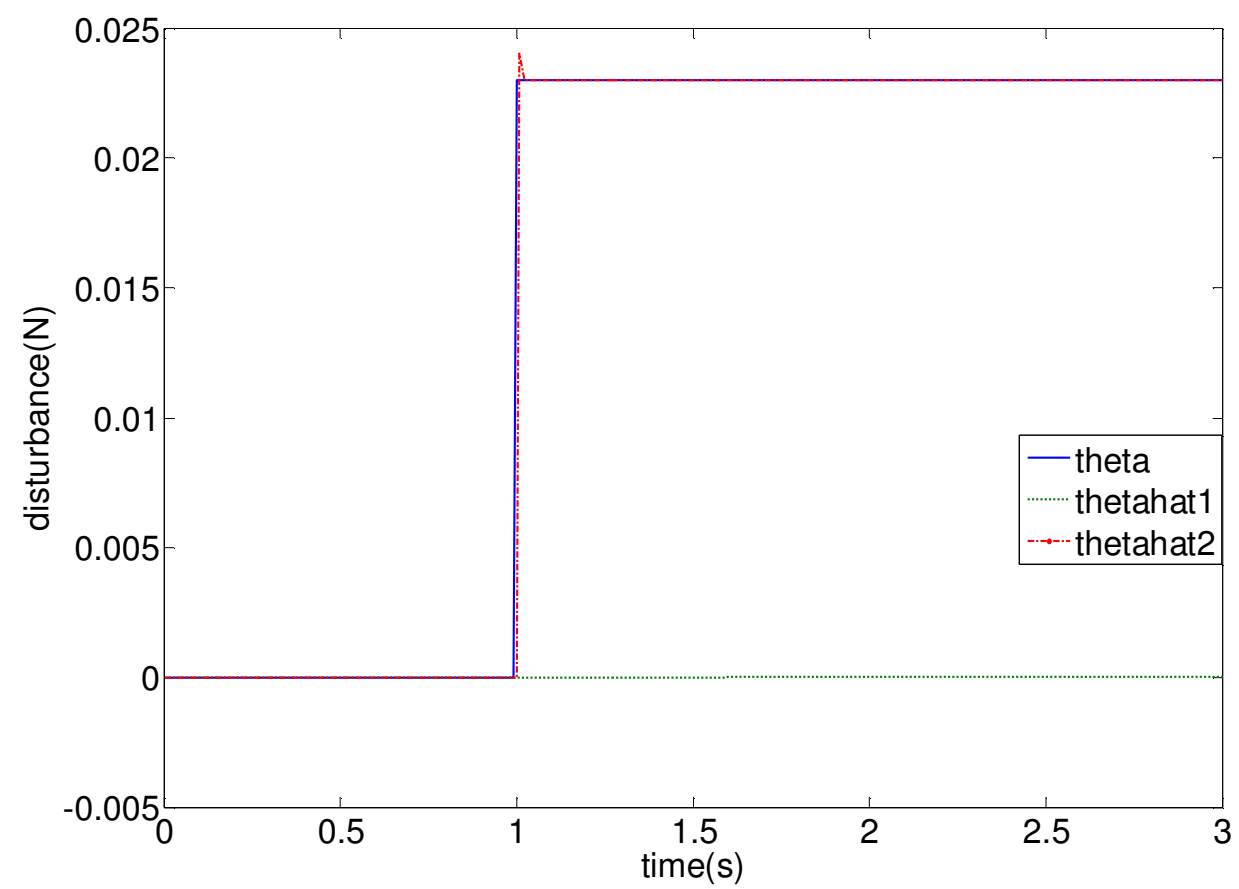

Figure 13: Disturbance and its estimates when $\gamma_{1}=10, \gamma_{2}=1$ 


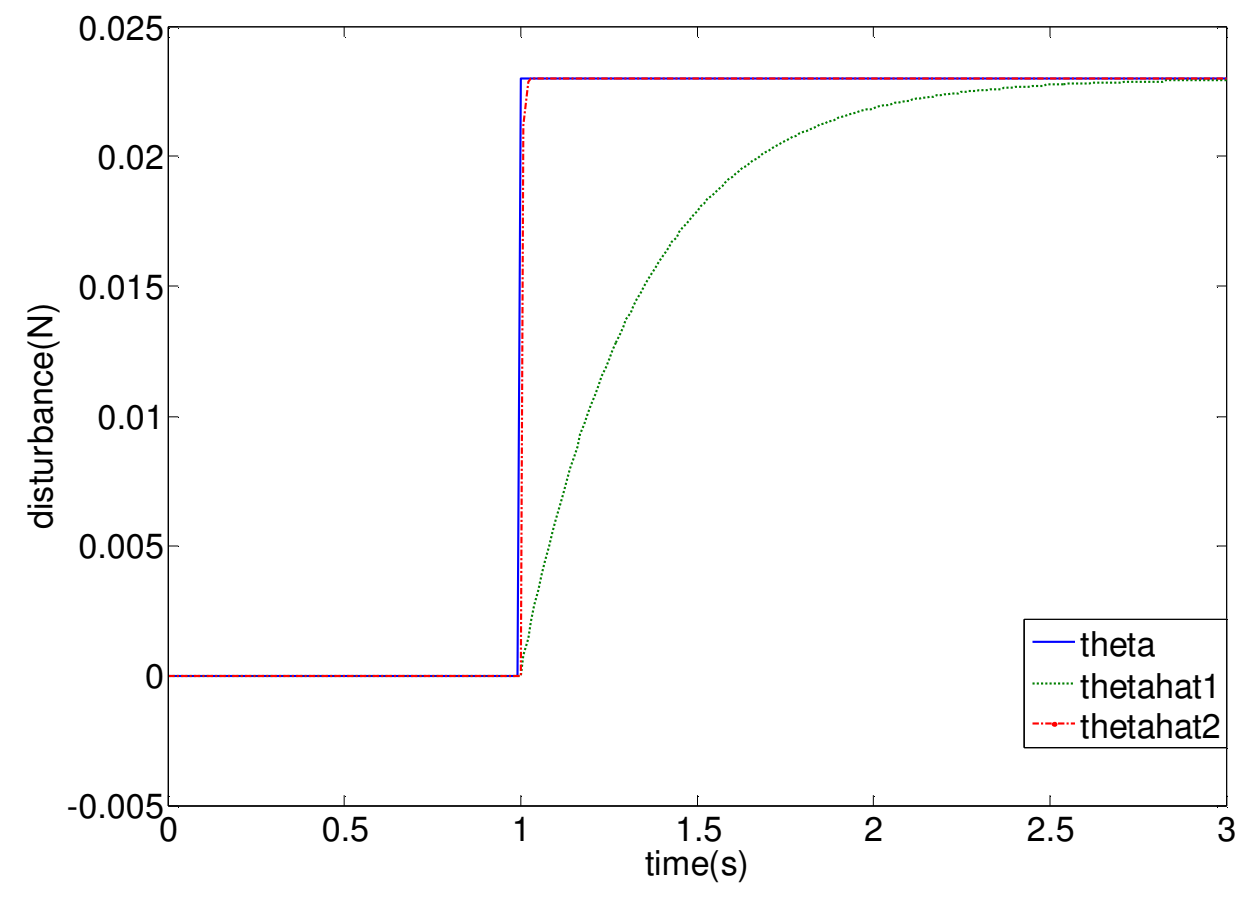

Figure 14: Disturbance and its estimates when $\gamma_{1}=2000, \gamma_{2}=0.1$

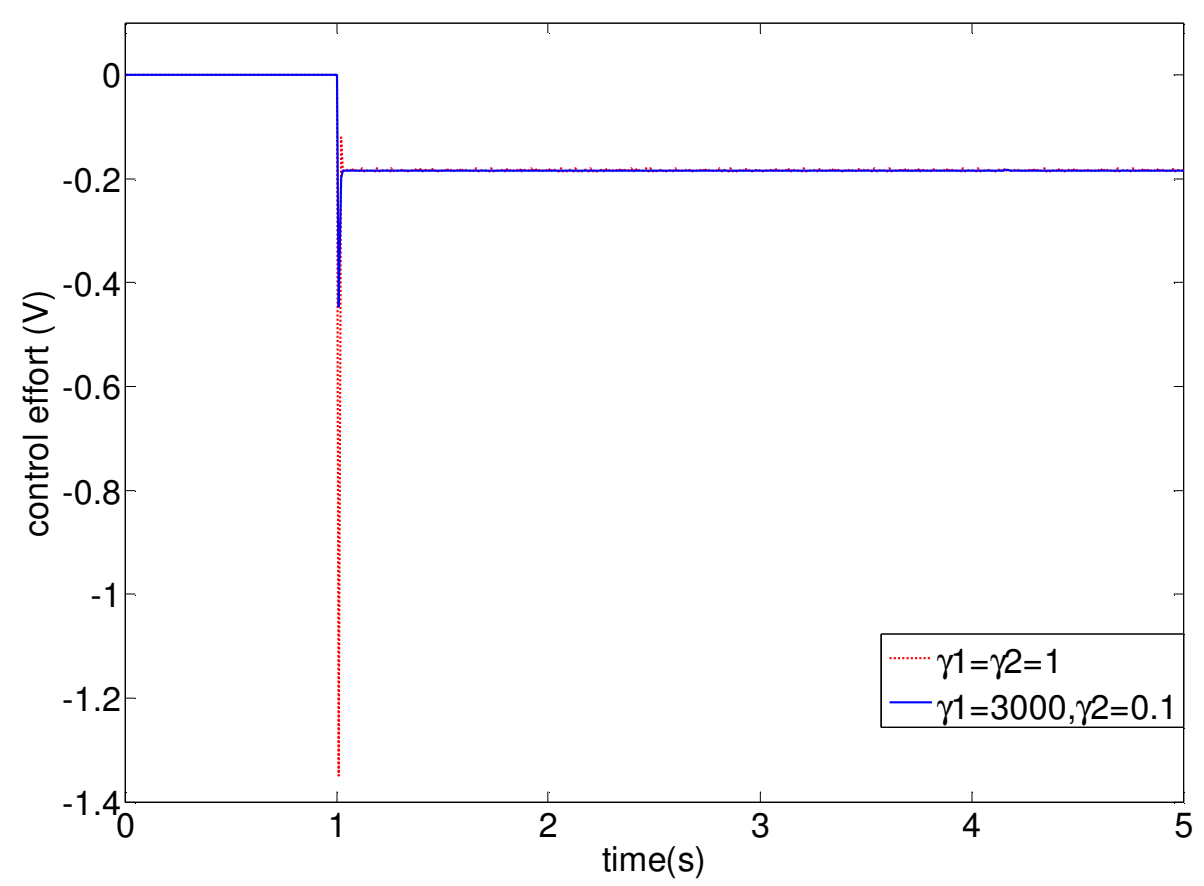

Figure 15: Control efforts with different AC 
Figure 12 through Figure 15 demonstrate that the responding speed of disturbance estimator relies on the value of ACs. Increasing the ACs value can drive the estimates of disturbance to the real one much faster. As a consequence, the settling time of the displacement $x_{1}$ responding to the disturbance is reduced, and $x_{1}$ is limited within 0.7 $\mathrm{mm}$. This can also be seen from the previous adaptation laws that the converging times and ACs are linear related respectively. In addition, the disturbance load is successfully estimated by two adaptation laws. However, in Figure 15, the control effort changes rapidly corresponding to a step disturbance. In reality, the disturbance will be gradually increased. So the rate of the change for control effort will be reduced accordingly as shown in Figure 11.

As shown from Figure 7 through Figure 15, the displacement of the rotor in AMB has been successfully controlled to almost zero without steady-state error by the ABC with different LCs and ACs. The adaptive laws estimate disturbance accurately. When choosing different LCs to achieve better performance, Lyapunov direct method guarantees a bounded value for each part in CLF at all time, and it drives the first state variable (displacement) to zero even when $\dot{V}=0$. Comparing the two sets of the results with different coefficients, we can see that the LCs play an important role in system's response. The larger the LCs values are, the smaller the overshoot values are. However, increasing LCs could lead to oscillations in control effort. It is also observed that if LCs are too small, transient responds for disturbance will oscillate significantly when the disturbance occurs. Adaptive coefficients (ACs) are in charge of the response time to the disturbance load. Increasing ACs can amplify the adaptation signals so that the estimation 
errors will converge to zeros faster. The simulation results also imply that even if the adaptation is turned off, that is when $\gamma_{i}=0(i=1,2)$, the states of the system are bounded as well, though there is a steady-state error in $x_{1}$.

\section{5 Robustness of $\mathrm{ABC}$}

One of the most important features of the adaptive control method is robustness. The uncertainties in the system parameter of $\mathrm{AMB}$ could be caused by the external forces such as a moving base or earthquake, or inner characteristic change due to temperature variations and the fatigue of the materials. If the closed-loop system has high robustness, those uncertainties should be tolerated in a certain margin. In the design process above, we can conclude that adaptation law estimates the disturbance no matter how big the disturbance is. However the bigger the disturbance is, the larger the spike value of $x_{1}$ becomes. A simulation under an extraordinary load disturbance of 450,000 $\mathrm{N}$ still shows that the peak position displacement remains within $0.0007 \mathrm{~m}$, the system is very robust to the disturbance [2]. Unfortunately, the overshoot of the control effort becomes unacceptable. Adding a saturation block in front of the control signal will destroy the stability of the system.

In the following simulation, ABC's robustness is tested by changing the system's parameters $(a, b, c, d)$, which are defined in (19), (20) and (21), by 200 and 1/200 times from their original values. We also increase the variation to the maximum in our simulation till the system becomes unstable to find the tolerance of the system to 
uncertainties. Figure 16 demonstrates the three states when varying parameter $a$ to 200 and $1 / 200$ of its actual value under the condition of. The figure shows the convergence of the displacement to zero in the presence of parameter variation.

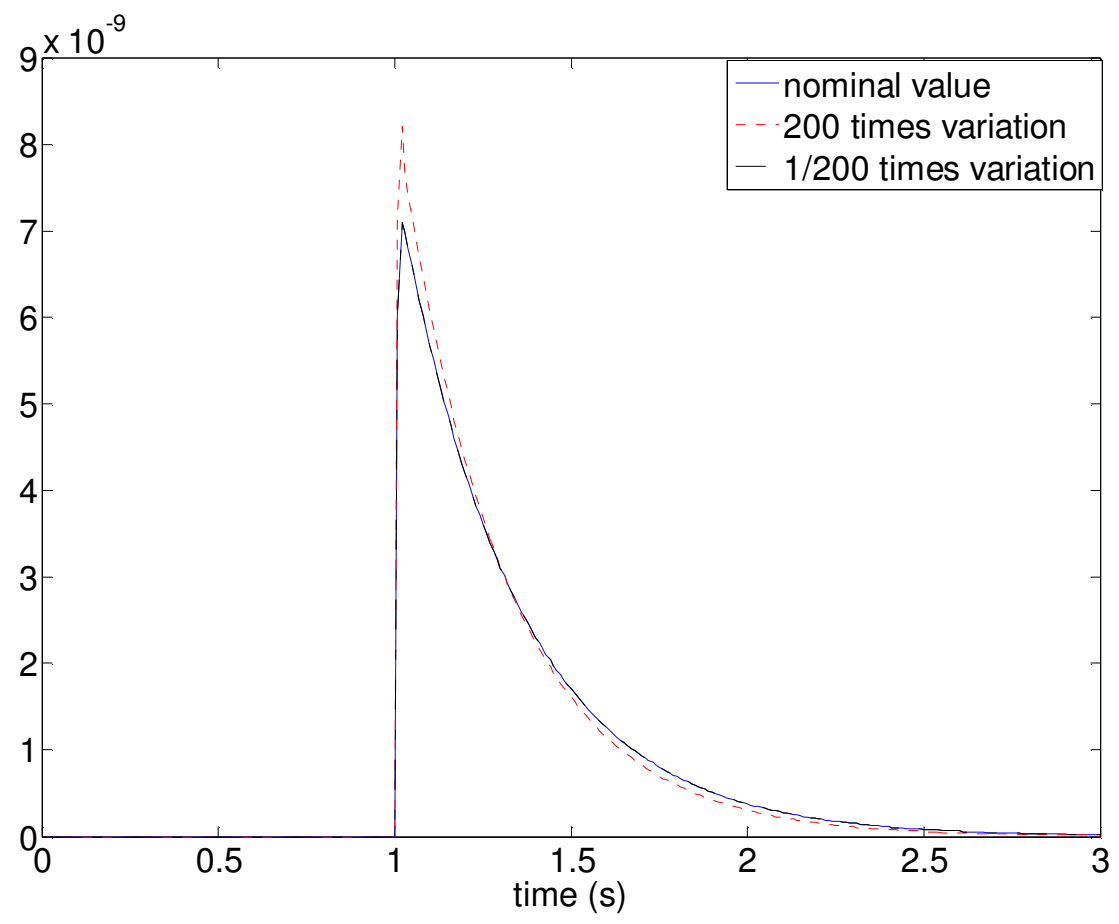

Figure 16: Position displacement when varying the parameter a to 200 and 1/200 times its original value

Table 2 lists the maximum overshoot values when the parameters vary 200 times of their original values. 
Table 2: Position of AMB with four parameters at a floating rate of $20000 \%$

\begin{tabular}{|c|c|c|}
\hline & $\begin{array}{c}\text { Peak Position (m) with } \\
20000 \% \text { Parameter } \\
\text { Value }\end{array}$ & $\begin{array}{c}\text { Peak Position (m) with } \\
-20000 \% \text { Parameter } \\
\text { Value }\end{array}$ \\
\hline$a$ & $8.21 \times 10^{-9}$ & $7.092 \times 10^{-9}$ \\
\hline$b$ & $8.216 \times 10^{-9}$ & $7.431 \times 10^{-9}$ \\
\hline$c$ & $7.434 \times 10^{-9}$ & $7.087 \times 10^{-9}$ \\
\hline$d$ & Unstable & $7.105 \times 10^{-9}$ \\
\hline
\end{tabular}

When the parameters are at their original values, the peak value of the position displacement is $7.094 \times 10^{-9} \mathrm{~m}$. In the simulation results, position is still bounded within the limitation and driven to zero while the settling time and overshoot value nearly the same as those with original parameter value. Table 3 shows the maximum factors by which system parameter can increase before the onset of instability.

Table 3: Maximum factors by which system parameter can increase before the onset of instability

\begin{tabular}{|c|c|c|c|}
\hline$a$ & $b$ & $c$ & $d$ \\
\hline 790 & 110000 & 110000 & 117 \\
\hline
\end{tabular}

This test shows that the $\mathrm{ABC}$ has a very tolerant capability for the uncertainty of every parameter that may change in reality. When it goes to the extreme situations that cause 
unstable control, $a$ should be at least 790 times of its original value and other parameters should be as large as listed in the table. It is worthwhile to mention that decreasing $c_{i}$ will weaken this robustness since smaller LCs generates bigger pike value of rotor displacement.

\section{6 Comparison with PID control}

The block diagram of a closed-loop PID controlled AMB system is shown in Figure 17.

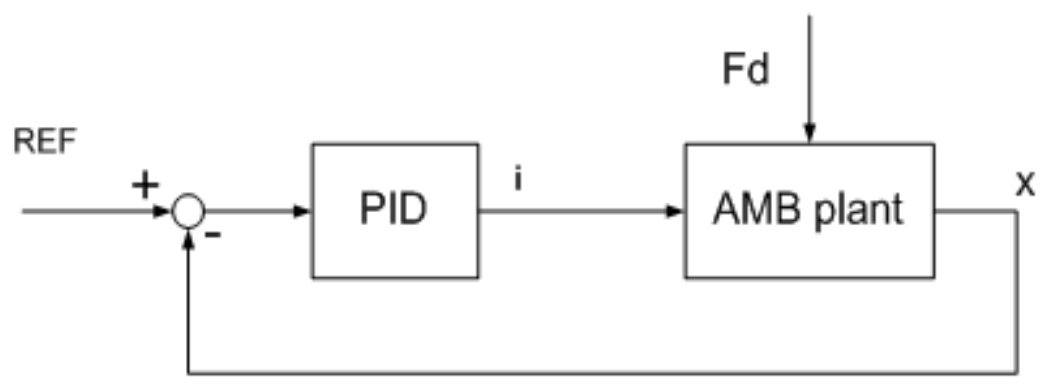

Figure 17: PID control of AMB with position feedback and current control

The PID controller has an input of the position error and its output is the current. The controller's optimized gains are defined in [4] and given in Table 4.

Table 4: Optimized PID gains [4]

\begin{tabular}{|c|c|}
\hline PID Gain & Value \\
\hline $\mathrm{K}_{\mathrm{P}}$ & 12258 \\
\hline $\mathrm{K}_{\mathrm{I}}$ & 2303 \\
\hline $\mathrm{K}_{\mathrm{D}}$ & 250 \\
\hline
\end{tabular}


The comparison of PID and $\mathrm{ABC}$ is represented by simulations as following in terms of performance and control effort when the disturbance is added to the system at $1 \mathrm{~s}$. The Lyapunov settings for $\mathrm{ABC}$ are $c_{1}=50, c_{2}=50, c_{3}=50, \gamma_{1}=100, \gamma_{2}=10$. These settings will result in a worse performance than that of Section III.4. The simulation results for both PID and ABC are shown in Figure 18 and Figure 19.

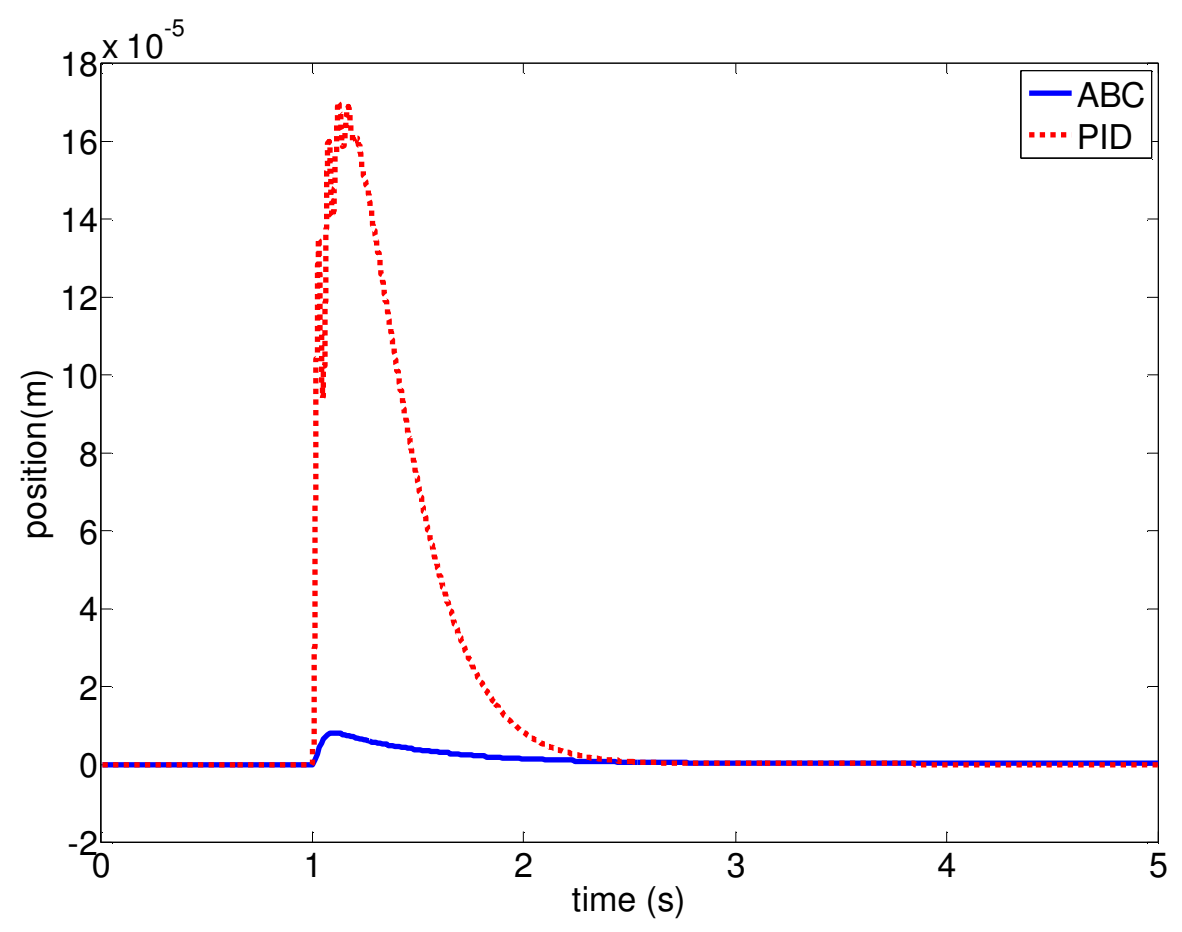

Figure 18: Position responses of $A B C$ and PID controlled $A M B$ 


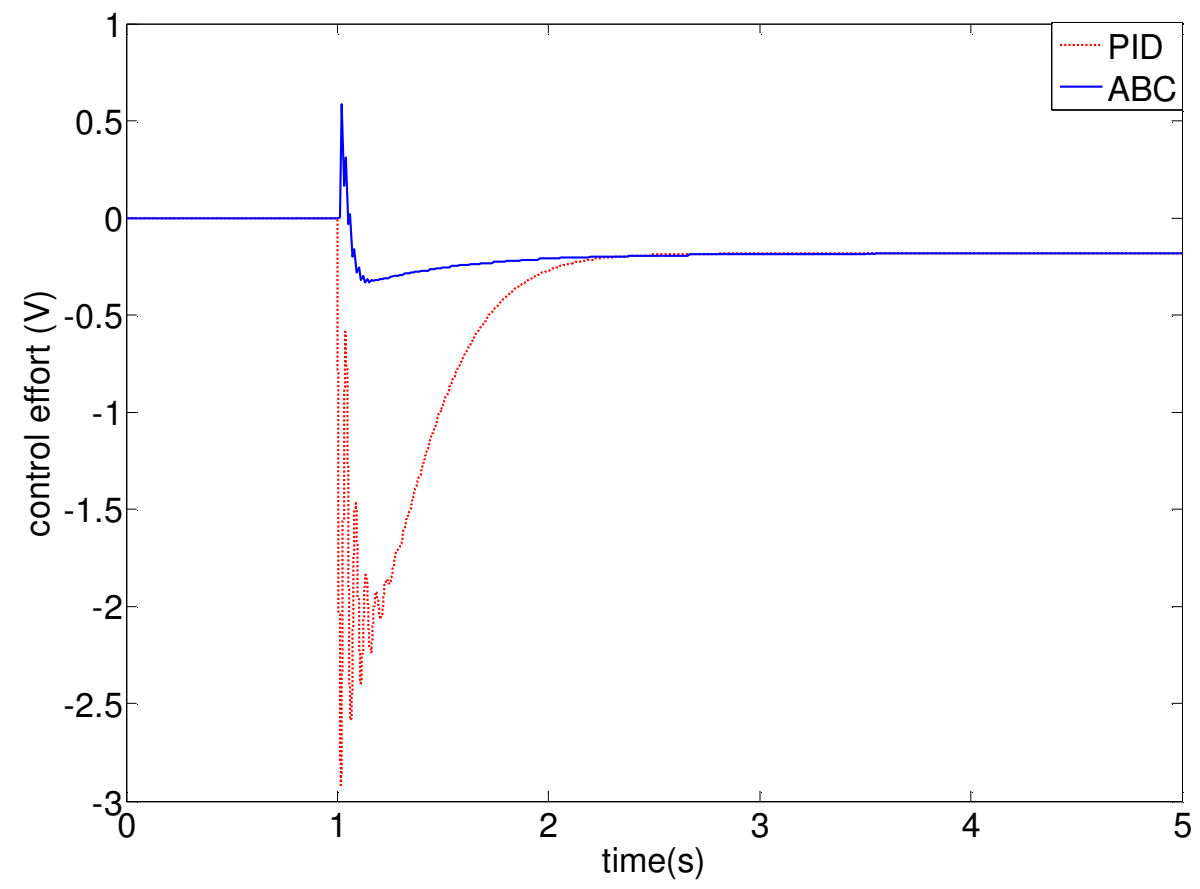

Figure 19: Control efforts of $A B C$ and PID controlled $A M B$

From Figure 18 and Figure 19, we can see that the results of $\mathrm{ABC}$ are better than that of PID control whose coefficients are optimized in [4]. The overshoot of the position in $\mathrm{ABC}$ is less than PID control and $\mathrm{ABC}$ has no oscillations. But the settling time is nearly the same for both controllers. Except for the oscillation at the very beginning of the onset of the displacement, the transient performance in control efforts in $\mathrm{ABC}$ is better than that of PID controller. There are several reasons why ABC performs better than PID. With the help of adaptive laws, external load is estimated on-line and compensated by control. However, in PID controller, proportional and derivative terms cause large overshoot and oscillation, meanwhile the use of integral term slows down the transient response. Nevertheless, the PID controller uses only one feedback that is position while the $\mathrm{ABC}$ is constructed based on three feedback signals (position, velocity, and current). 


\section{7 Summary of the chapter}

The ABC strategy is derived with the knowledge of three state variables. Simulation results validate the effectiveness of this control law. The system returns to its equilibrium point when $x_{1}=0$ in a short settling time with very small peak overshoot. Steady-state error converges to zero asymptotically. The variations of LCs and ACs can change the responding performance of the AMB system. However, the selection of these positive constants depends on the designer's experience. If they exceed some limitations, oscillation becomes obvious and control effort increases, and the most serious consequence is unstable control.

In addition, the robustness of the $\mathrm{ABC}$ controlled $\mathrm{AMB}$ system against parameter variations is tested and demonstrated in this chapter. The $\mathrm{ABC}$ also shows superior control performance to a PID control system. 


\section{CHAPTER IV}

\section{ADAPTIVE OBSERVER-BASED BACKSTEPPING CONTROL}

The results in chapter III are under the assumption that full state feedback is available. In this chapter, we remove the non-measurable states (velocity and current) and develop an AOBC design based on one measurable state that is position displacement. Meanwhile, the disturbance signal $\left(F_{d}\right)$ is also taken as the unknown force on the rotor.

\section{1 Control objective and strategy}

Our control objective is to design a control law to track reference position input with the position feedback of the plant. Figure 20 shows the typical structure of AOBC with an amplifier that will not be used in our design. 


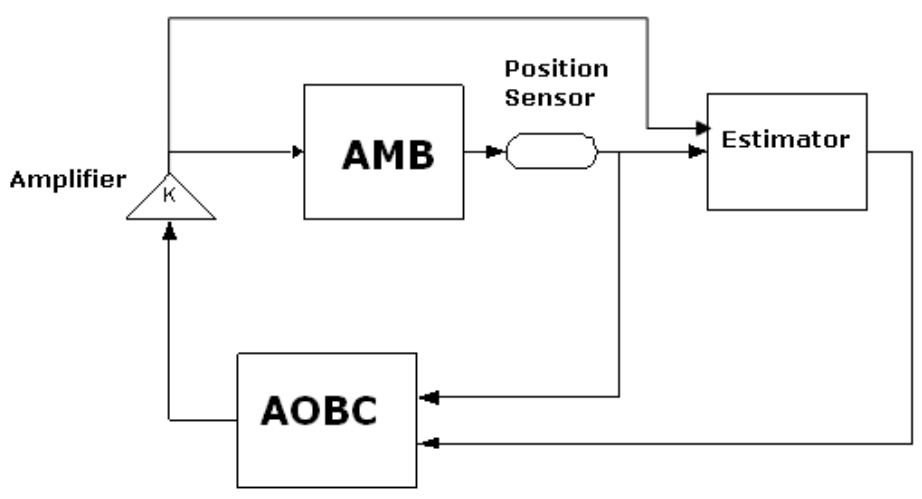

Figure 20: Model of AOBC applied AMB

Since the only information that can be measured is the position displacement, we need an exponentially convergent observer to estimate other unavailable states. The observation errors will be treated in the design of CLF by a special designed term called nonlinear damping. Based on the observed information, we can design the AOBC laws in the way introduced in Chapter III. In order to make the control system asymptotically stable and to regulate the error terms, CLF has to be constructed as in Figure 21.

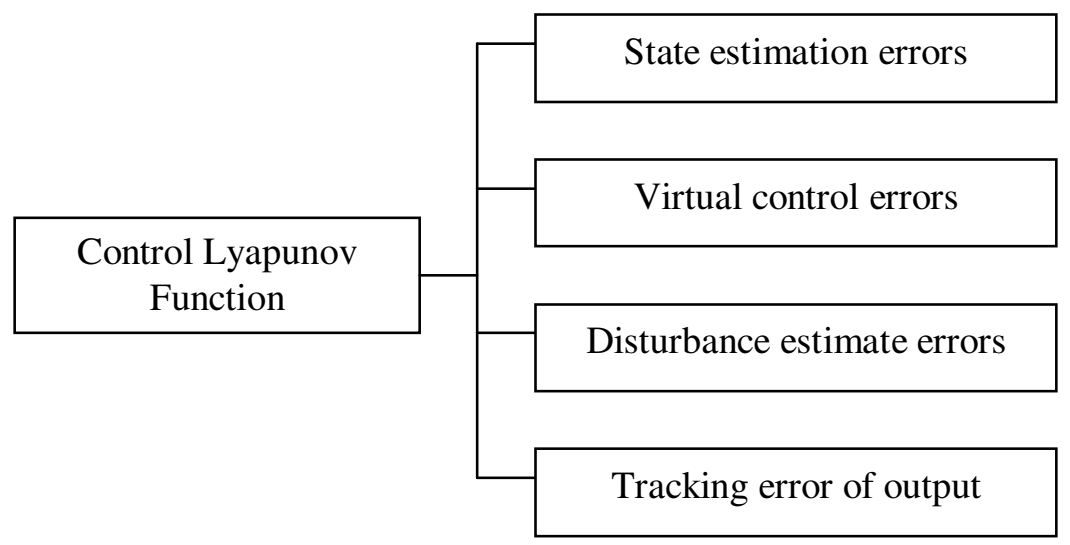

Figure 21: Construction of CLF in AOBC 
For applying the AOBC strategy to the AMB system, we have to transform the model of the ABM to output-feedback form [13] as shown in (41) for which the exponential observers are assumed to be available and the output is the displacement. The terms $\varphi_{i}(i$ $=1,2 \ldots n)$ and $\beta$ are linear and nonlinear functions of $y$ and $b_{i}(i=0,1,2 \ldots . . m)$ are constants.

$$
\begin{aligned}
& \dot{x}_{1}=x_{2}+\varphi_{1}(y) \\
& \dot{x}_{2}=x_{3}+\varphi_{2}(y) \\
& \vdots \\
& \dot{x}_{\rho}=x_{\rho+1}+\varphi_{\rho}(y)+b_{m} \beta(y) u \\
& \vdots \\
& \dot{x}_{n-1}=x_{n}+\varphi_{n-1}(y)+b_{1} \beta(y) u \\
& \dot{x}_{n}=\varphi_{n}(y)+b_{0} \beta(y) u \\
& y=x_{1}
\end{aligned}
$$

The system represented by (41) is assumed to be a minimum phase system, that means $b_{m} s^{m}+\cdots b_{1} s+b_{0}$ has to be a Hurwitz polynomial and $\beta(y) \neq 0$. This ensures the existence of an exponentially convergent observer. Another assumption is that the reference signal and its first derivatives are known, and they are bounded and piecewise continuous. These assumptions ensure that there exists a feedback control which could guarantee the global boundless of $x$ and $\hat{x}$.

\section{2 Model transformation}

In order to apply $\mathrm{AOBC}$ to the $\mathrm{AMB}$ system, we need the system to be transformed to the observable output feedback form. The transfer functions of the AMB with the respect to output $X(s)$ and disturbance $F_{d}(s)$ are: 


$$
\begin{aligned}
& \frac{X(s)}{U(s)}=\frac{2 k_{i}}{m L s^{3}+m R s^{2}+2\left(k_{i}^{2}-L k_{s}\right) s-2 k_{s} R} \\
& \frac{F_{d}(s)}{U(s)}=\frac{L s+R}{m L s^{3}+m R s^{2}+2\left(k_{i}^{2}-L k_{s}\right) s-2 k_{s} R}
\end{aligned}
$$

We can transform the system transfer functions (42) and (43) to the following output feedback form.

$$
\begin{gathered}
\dot{x}=\left[\begin{array}{ccc}
-\frac{R}{L} & 1 & 0 \\
\frac{-2\left(k_{i}^{2}-L k_{s}\right)}{m L} & 0 & 1 \\
\frac{2 k_{s} R}{m L} & 0 & 0
\end{array}\right] x+\left[\begin{array}{c}
0 \\
0 \\
\frac{2 k_{i}}{m L}
\end{array}\right] u+\left[\begin{array}{c}
0 \\
\frac{1}{m} \\
\frac{R}{m L}
\end{array}\right] F_{d} \\
y=x_{1}
\end{gathered}
$$

The first state variable in (44) is position and input is control effort $u$. However, due to the transformation, the second and third state variables are not the velocity and current anymore. Instead, they do not have physical meanings but are just used for the convenience of constructing the state equations in (44).

\section{3 Observer design}

We can rewrite (44) as

$$
\begin{aligned}
& \dot{x}_{1}=x_{2}+\varphi_{1}(y) \\
& \dot{x}_{2}=x_{3}+\varphi_{2}(y)+\theta \\
& \dot{x}_{3}=\varphi_{3}(y)+u^{\prime}+a \theta
\end{aligned}
$$


where $\quad \varphi_{1}(y)=-\frac{R}{L} x_{1} \quad, \quad \varphi_{2}(y)=\frac{-2\left(k_{i}^{2}-L k_{s}\right)}{m L} x_{1} \quad, \quad a=\frac{R}{L} \quad, \quad \varphi_{3}(y)=\frac{2 k_{s} R}{m L} x_{1} \quad$, $u^{\prime}=\frac{2 k_{i}}{m L} u, \theta=\frac{F_{d}}{m}=1$.

The state observer can be constructed as

$$
x=\underbrace{\xi_{0}+\theta \xi_{1}}_{\begin{array}{c}
\text { virtual control } \\
\text { candidates }
\end{array}}+\underset{\begin{array}{c}
\text { estimate } \\
\text { error }
\end{array}}{\varepsilon}
$$

where $x$ is a state vector, $\xi_{0}=\left[\begin{array}{lll}\xi_{01} & \xi_{02} & \zeta_{03}\end{array}\right]^{T}$, and $\xi_{1}=\left[\begin{array}{lll}\xi_{11} & \xi_{12} & \zeta_{13}\end{array}\right]^{T}$. The definitions of the vectors $\xi_{0}$ and $\xi_{1}$ are given as follows.

$$
\left\{\begin{array}{l}
\dot{\xi}_{01}=k_{1}\left(y-\xi_{01}\right)+\xi_{02}+\varphi_{1}(y) \\
\dot{\xi}_{02}=k_{2}\left(y-\xi_{01}\right)+\xi_{03}+\varphi_{2}(y) \\
\dot{\xi}_{03}=k_{3}\left(y-\xi_{01}\right)+u^{\prime}+\varphi_{3}(y)
\end{array}\right.
$$

and

$$
\left\{\begin{array}{l}
\dot{\xi}_{11}=-k_{1} \xi_{11}+\xi_{12} \\
\dot{\xi}_{12}=-k_{2} \xi_{12}+\xi_{13}+1 \\
\dot{\xi}_{13}=-k_{3} \xi_{13}+a
\end{array}\right.
$$

Equation (47) shows the filters without disturbance. Equation (48) describes the filters including disturbance. The constants $k_{i}(i=1,2,3)$ are chosen as following.

We set a matrix as $A_{0}=\left[\begin{array}{ccc}-k_{1} & 1 & 0 \\ -k_{2} & 0 & 1 \\ -k_{3} & 0 & 0\end{array}\right]$ and choose gain vector $\left[\begin{array}{lll}k_{1} & k_{2} & k_{3}\end{array}\right]^{T}$ to make $A_{0}$

Hurwitz. Then the system can be represented by 


$$
\dot{x}=A_{0} x+k y+\varphi(y)+\left[\begin{array}{lll}
0 & 1 & a
\end{array}\right]^{T} \theta+\left[\begin{array}{lll}
0 & 0 & 1
\end{array}\right]^{T} u^{\prime}
$$

The two filters become

$$
\begin{gathered}
\dot{\xi}_{0}=A \xi_{0}+k y+\varphi(y)+\left[\begin{array}{lll}
0 & 0 & 1
\end{array}\right]^{T} u^{\prime} \\
\dot{\xi}_{1}=A_{0} \xi_{1}+\left[\begin{array}{lll}
0 & 1 & a
\end{array}\right]^{T}
\end{gathered}
$$

Given (49), (50) and (51), we have the observer error $\dot{\varepsilon}=\dot{x}-\dot{\xi}_{0}-\dot{\xi}_{1}=A_{0}\left(x-\xi_{0}-\xi_{1} \theta\right)=A_{0} \varepsilon$. Since $A_{0}$ is a Hurwitz matrix, the estimation errors of the states which are observed by this observer will exponentially decay.

\section{4 Design procedure of the control law}

In this section, we develop the control law in a similar way to the one in Chapter III. We also need to take estimator errors into consideration. The special tool we use to counteract the observer error is nonlinear damping, which strengthens the negativity of the derivative of the CLF by adding a negative square term. Our control goal is to make output $y$ zero. At first, like we have done in Chapter III, the first state of the error system is chosen as

$$
z_{1}=y
$$

The derivative of $z_{1}$ is

$$
\dot{z}_{1}=x_{2}+\varphi_{1}(y)
$$


Since $x_{2}$ is non-measurable, we replace $x_{2}$ with the observed state of it in (46). Then we will have

$$
x_{2}=\xi_{02}+\xi_{12} \theta+\varepsilon_{2}
$$

Substituting (54) into (53) to obtain

$$
\dot{z}_{1}=\xi_{02}+\xi_{12} \theta+\varepsilon_{2}+\varphi_{1}(y)
$$

Then the product of $z_{1}$ and $\dot{z}_{1}$ will be

$$
z_{1} \dot{z}_{1}=z_{1}\left(\xi_{02}+\xi_{12} \theta+\varepsilon_{2}+\varphi_{1}(y)\right)
$$

Now we need to choose the virtual control from the above known variables. Since only $\xi_{0}$ contains the control $u^{\prime}, \xi_{01}$ is the virtual control, so the stabilization function to replace $\xi_{01}$ will be

$$
\alpha_{1}=-c_{1} z_{1}-d_{1} z_{1}-\xi_{12} \theta_{1}+\varphi_{1}(y)
$$

where $\theta_{1}$ is the first estimate of the disturbance. Set the CLF for the first equation of (45); all the terms which need to be driven to zero should be included.

$$
V_{1}=\frac{1}{2} z_{1}^{2}+\frac{1}{2 d_{1}} \varepsilon^{T} P_{0} \varepsilon+\frac{1}{2 \gamma_{1}} \tilde{\theta}_{1}^{2}
$$

This is different from (22) by the term $\frac{1}{2 d_{1}} \varepsilon^{T} P_{0} \varepsilon$, in which $\varepsilon$ represents the observer error which needs to be compensated by nonlinear damping. 
In (58), $P_{0}$ is a positive definite and symmetric matrix. If we define $A_{0}^{T} P_{0}+P_{0} A_{0}=-Q$, where $A_{0}$ is defined in our estimator error system $\dot{\varepsilon}=A_{0} \varepsilon$, then this system's derivative of CLF $V=\varepsilon^{T} P_{0} \varepsilon$ will be

$$
\begin{aligned}
\dot{V} & =\dot{\mathcal{\varepsilon}}^{T} P_{0} \varepsilon+\varepsilon^{T} P_{0} \dot{\varepsilon} \\
& =\varepsilon^{T}\left(A_{0}^{T} P_{0}+P_{0} A_{0}\right) \mathcal{\varepsilon} \\
& =-\varepsilon^{T} Q \varepsilon
\end{aligned}
$$

In our case, matrix $Q=I$, then $\dot{V}=-\varepsilon^{T} \varepsilon$. We will use this result along with a nonlinear damping to counteract the estimation error affect.

$$
z_{1} \dot{z}_{1}=-c_{1} z_{1}^{2}-d_{1} z_{1}^{2}+z_{1} \xi_{12} \tilde{\theta}_{1}+z_{1} z_{2}+z_{1} \varepsilon_{2}
$$

It should be noticed that there is a new term $-d_{1} z_{1}^{2}$ appears in the above equation. This term is designed to cooperate with the derivative of $\frac{1}{2 d_{1}} \varepsilon^{T} P_{0} \varepsilon$ and $z_{1} \varepsilon_{2}$ to make up a quadratic negative term shown in (61). Substituting (60) into $\dot{V}$ to obtain

$$
\dot{V}_{1}=-c_{1} z_{1}^{2}-d_{1} z_{1}^{2}+z_{1} z_{2}+\tilde{\theta}_{1}\left(z_{1} \xi_{12}-\frac{\dot{\hat{\theta}}_{1}}{\gamma_{1}}\right)-\left(\sqrt{d_{1} z_{1}}-\frac{1}{2 \sqrt{d_{1}}} \varepsilon_{2}\right)^{2}+\frac{1}{4 d_{1}} \varepsilon_{2}^{2}-\frac{1}{d_{1}} \varepsilon^{2}
$$

As we expounded in Chapter III, the term $z_{1} z_{2}$ in (61) will be eliminated later by $\dot{\alpha}_{2}$, if only the adaptive update law of first estimator is selected as

$$
\dot{\hat{\theta}}_{1}=\gamma_{1} z_{1} \xi_{12}
$$

then $\dot{V}_{1} \leq 0$. Let's consider the second equation in (45). 


$$
\begin{aligned}
\dot{z}_{2} & =\dot{\xi}_{02}-\dot{\alpha}_{1} \\
& =\xi_{03}+\varphi_{2}(y)+k_{2}\left(y-\xi_{01}\right)-\frac{\partial \alpha_{1}}{\partial z_{1}}\left(\xi_{02}+\xi_{12} \theta+\varepsilon_{2}+\varphi_{1}(y)\right)-\frac{\partial \alpha_{1}}{\partial \theta_{1}} \dot{\theta}_{1}-\frac{\partial \alpha_{1}}{\partial \xi_{12}}
\end{aligned}
$$

The second stabilizing function is chosen as

$$
\begin{aligned}
\alpha_{2}= & -z_{1}-c_{2} z_{2}-d_{2}\left(\frac{\partial \alpha_{1}}{\partial z_{1}}\right)^{2} z_{2}-\varphi_{2}(y)-k_{2}\left(y-\xi_{01}\right) \\
& +\frac{\partial \alpha_{1}}{\partial z_{1}}\left(\xi_{02}+\xi_{12} \hat{\theta}_{2}+\varphi_{1}(y)\right)+\frac{\partial \alpha_{1}}{\partial \theta_{1}} \dot{\theta}_{1}+\frac{\partial \alpha_{1}}{\partial \xi_{12}} \dot{\xi}_{12}
\end{aligned}
$$

so that

$$
z_{2} \dot{z}_{2}=z_{2} z_{3}+z_{2}\left(c_{1}+d_{1}+\varphi_{1}\right) \xi_{12} \tilde{\theta}_{2}+\left(c_{1}+d_{1}+\varphi_{1}\right) \varepsilon_{2} z_{2}-c_{2} z_{2}^{2}-d_{2} z_{2}^{2}\left(\frac{\partial \alpha_{1}}{\partial z_{1}}\right)^{2} z_{2}^{2}
$$

We construct the second CLF as

$$
V_{2}=V_{1}+\frac{1}{2} z_{2}^{2}+\frac{1}{d_{2}} \varepsilon^{T} P_{0} \varepsilon+\frac{1}{2 \gamma_{2}} \tilde{\theta}_{2}^{2}
$$

Given (65), the derivative of second CLF becomes

$$
\dot{V}_{2}=\dot{V}_{1}-c_{2} z_{2}^{2}-\left(\sqrt{d_{2}} \frac{\partial \alpha_{1}}{\partial z_{1}} z_{2}-\frac{1}{2 \sqrt{d_{2}} \varepsilon_{2}}\right)^{2}+\frac{1}{4 d_{2}} \varepsilon_{2}^{2}-\frac{1}{d_{2}} \varepsilon^{2}
$$

If the adaptive updated law of the second estimator is

$$
\dot{\hat{\theta}}_{2}=-\gamma_{2} z_{2}\left(\frac{\partial \alpha_{1}}{\partial z_{1}}\right) \xi_{12}
$$

the final CLF will be set as 


$$
V_{3}=V_{1}+V_{2}+\frac{1}{2} z_{3}^{2}+\frac{1}{d_{3}} \varepsilon^{T} P_{0} \varepsilon+\frac{1}{2 \gamma_{3}} \tilde{\theta}_{3}^{2}
$$

whose derivative includes

$$
z_{3} \dot{z}_{3}=z_{3}\left(\varphi_{3}(y)+u^{\prime}+k_{3}\left(y-\xi_{01}\right)-\dot{\alpha}_{2}\right)
$$

If the control law is designed as

$$
u^{\prime}=-c_{3} z_{3}-d_{3}\left(\frac{\partial \alpha_{2}}{\partial z_{1}}\right)^{2} z_{3}-z_{2}-\varphi_{3}(y)-k_{3}\left(y-\xi_{01}\right)+\dot{\alpha}_{2}
$$

the derivative of the CLF will be

$$
\dot{V}_{3}=\dot{V}_{1}+\dot{V}_{2}-c_{3} z_{3}^{2}-\left(\sqrt{d_{3}} \frac{\partial \alpha_{2}}{\partial z_{1}} z_{3}-\frac{1}{2 \sqrt{d_{3}} \varepsilon_{3}}\right)^{2}-\left(\frac{\partial \alpha_{2}}{\partial z_{1}} z_{3} \xi_{12}+\frac{\hat{\theta}_{3}}{\gamma_{3}}\right)+\frac{1}{4 d_{3}} \varepsilon_{3}^{2}-\frac{1}{d_{3}} \varepsilon^{2}
$$

Therefore, we design the third disturbance estimator as

$$
\dot{\hat{\theta}}_{3}=-\gamma_{3} \frac{\partial \alpha_{2}}{\partial y} \xi_{12} z_{3}
$$

Substituting (73) into (72), we will have $\dot{V}_{3} \leq 0$. The control goal will be achieved by applying $u^{\prime}$ derived in (71) to (44).

\section{5 Simulation results and analysis}

The block diagram in Figure 22 is constructed based on the control law developed above. The subsystem "plant" contains the original AMB model and its observer, and the input 
of the plant is the control law constructed by the AOBC. The output of the plant is the position displacement. The reference $r$ is set to be zero.

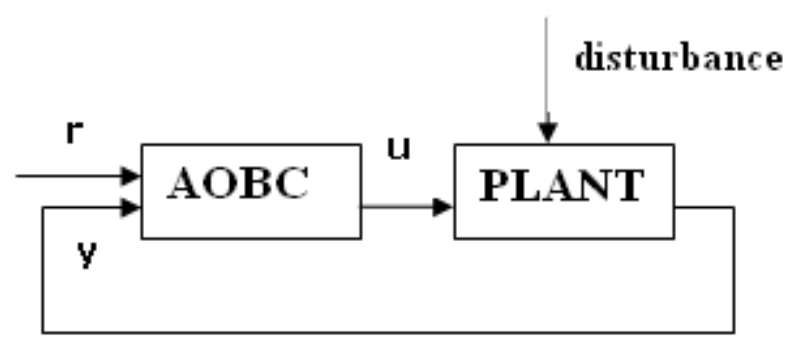

Figure 22: Block diagram of $A O B C$

The following figures show the rotor displacement, disturbance estimation and control efforts of the closed-loop AMB system. In the parameter settings, the measured disturbance in Matlab should be 1 because of the transformation from the original system to the output feedback form. The step time of the disturbance begins at $1 \mathrm{~s}$ and the Lyapunov coefficients settings are: $c_{1}=5000, c_{2}=1000, c_{3}=50$, $\gamma_{1}=15000, \gamma_{2}=100, \gamma_{3}=1$, these are selected based on the experience in previous chapter. The nonlinear damping coefficients are chosen as $d_{1}=d_{2}=d_{3}=1 e^{-5}$. 


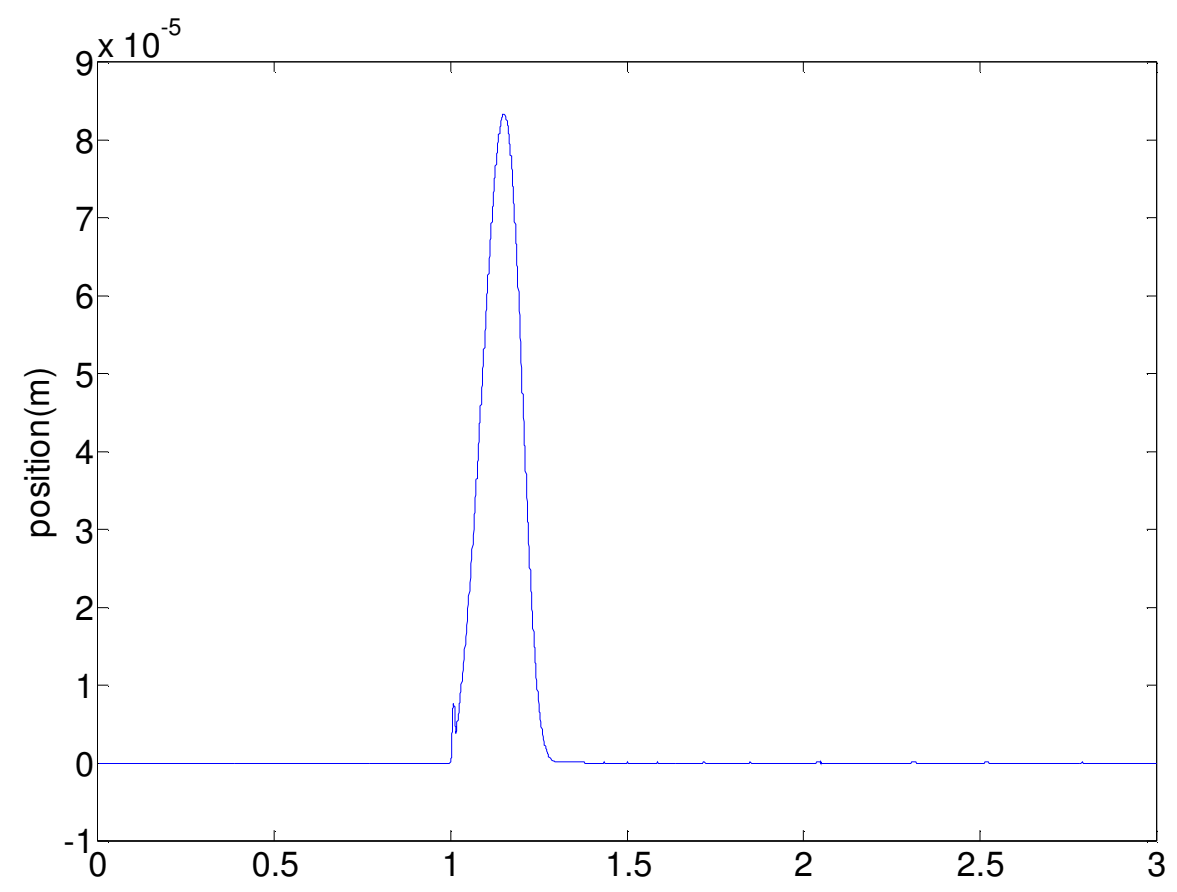

Figure 23: Position displacement under the control of $A O B C$

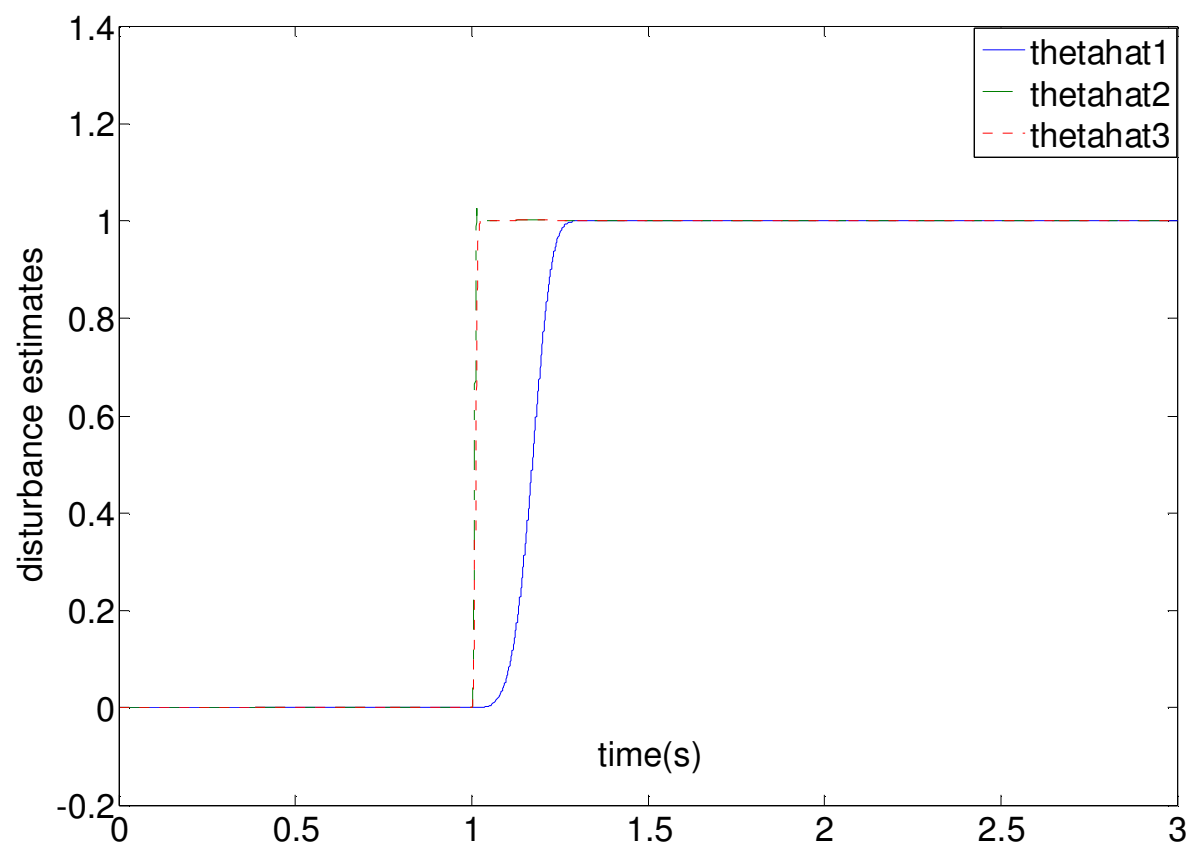

Figure 24: Disturbance estimates by three adaptive laws 


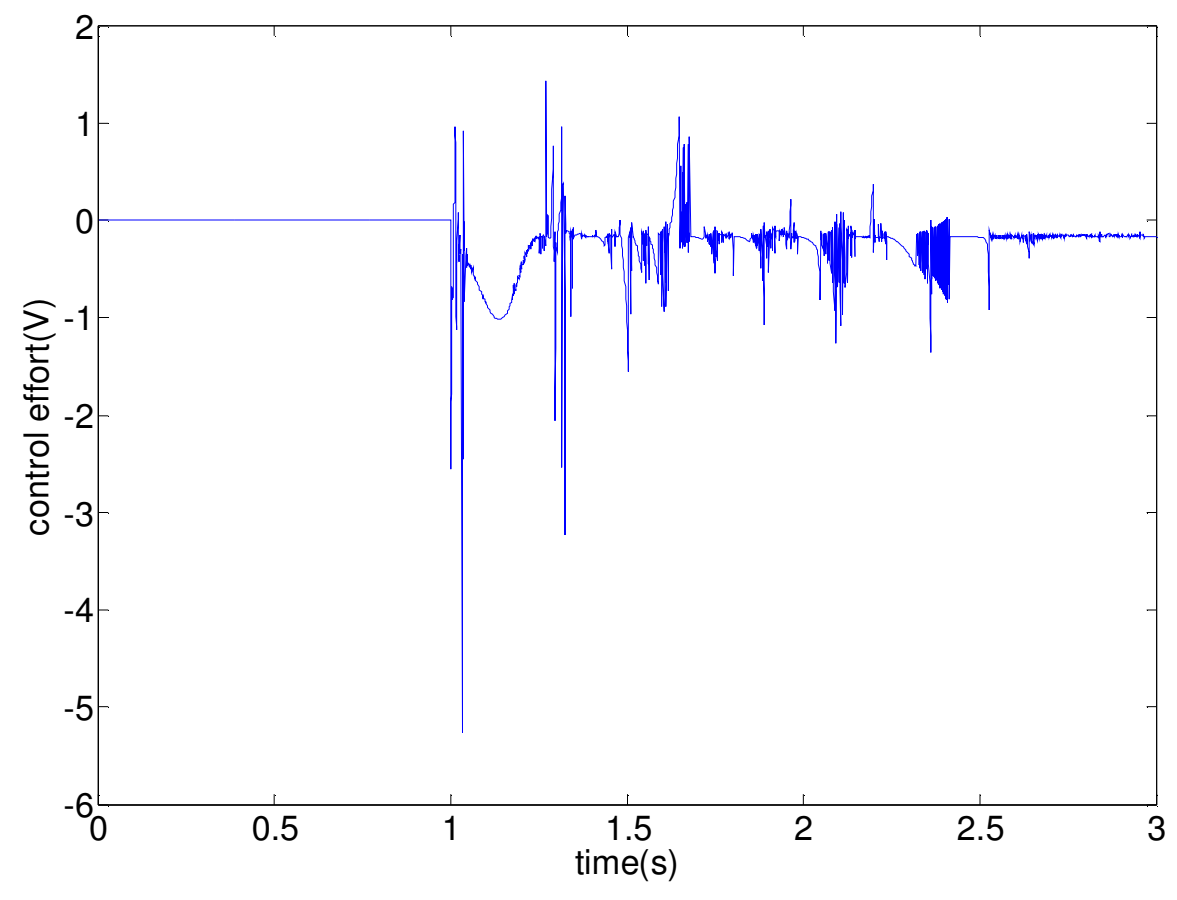

Figure 25: Control effort of $A O B C$

Figure 23 and Figure 24 illustrate that the control objective is achieved by AOBC. The position of the rotor is driven to its equilibrium point with its peak value within the limitation of the air gap width. The three adaptive updated laws successfully estimate the disturbance. Because the other two state variables have been changed by a matrix transformation, it is no need to measure them as long as the position is still $x_{1}$. The ways of tuning Lyapunov coefficients also affects control results in AOBC, it acts in a similar way as well as $\mathrm{ABC}$. The noisy oscillations in Figure 25 are caused by the large Lyapunov coefficients and can be improved by reducing the values of LCs and ACs. However, the position overshoot will grow as the values of LCs and ACs are increased. 
The overshoot and settling time of the position response with respect to different coefficients LCs and ACs are shown in Table 5. The LCs and ACs are increased separately.

Table 5: Disturbance response characteristics in position with different Lyapunov coefficients

\begin{tabular}{|c|l|l|c|}
\hline & $\begin{array}{l}c_{1}=c_{2}=c_{3}=500 \\
\gamma_{1}=\gamma_{2}=100 \\
\gamma_{3}=1\end{array}$ & $\begin{array}{l}c_{1}=5000, c_{2}=1000 \\
c_{3}=50, \gamma_{1}=\gamma_{2}=100 \\
\gamma_{3}=1\end{array}$ & $\begin{array}{c}c_{1}=5000, c_{2}=1000 \\
c_{3}=500, \gamma_{1}=150000 \\
\gamma_{2}=100, \gamma_{3}=1\end{array}$ \\
\hline Peak value(m) & $5.87 \times 10^{-3}$ & $1.34 \times 10^{-4}$ & $8.32 \times 10^{-5}$ \\
\hline Settling time(s) & 0.87 & 1.88 & 0.31 \\
\hline
\end{tabular}

From Table 5, we can see that all of the three sets of simulations achieve the control goal. However, by comparing the performance of the $\mathrm{AOBC}$ with that of $\mathrm{ABC}$ in Chapter III, we can observe that the overshoot value of the position response for AOBC is much larger than the one for $\mathrm{ABC}$. The control effort of $\mathrm{ABC}$ is smooth only except for a sharp overshoot at the time instant when disturbance changes while the control effort of AOBC oscillates unpredictably in Figure 25. The decrease of the control performance of AOBC is because the only available information of the original system is the position signal. We have to increase the Lyapunov coefficients to achieve a better control result.

\section{6 Robustness of AOBC}

From the adaptive update law deviation for the disturbance, AOBC has a high capability of disturbance rejection. To make the peak of $x_{1}$ pass its limitation, the disturbance 
should be increased by 9.6 times, which means the external load could be $44.2 \mathrm{~N}$ while the system is still stable. By changing the value of the parameter $\varphi_{3}$ to 2.3 times and 1/2.3 times of its original value, we test the robustness of AOBC when $c_{1}=5000, c_{2}=1000, c_{3}=50, \gamma_{1}=150000, \gamma_{2}=100, \gamma_{3}=1$ without tuning the observer parameters and control law. The position responses are shown in Figure 26.

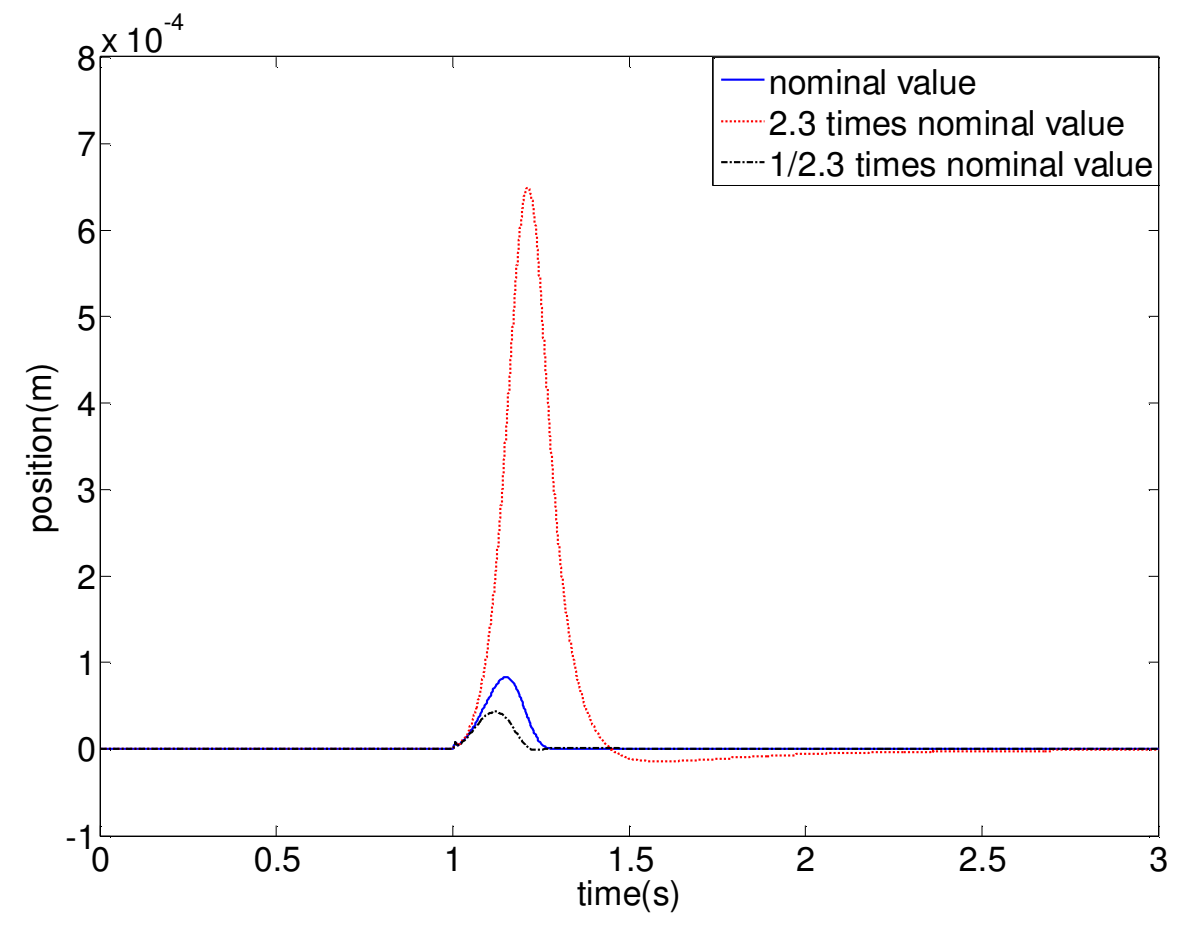

Figure 26: Position responses when $\varphi_{3}$ varies

If the parameters do not change, the peak value of position displacement is $8.32 \times 10^{-5} \mathrm{~m}$. The figure above shows that with the parameter $\varphi_{3}$ varying from its original value to $230 \%$ of its original value, the displacement is still in the air gap limit. If we increase $\varphi_{3}$ by over 2.3 times, or increase $\varphi_{1}$ and $\varphi_{2}$ by over 2.3 times, the system will be unstable or the peak value of the rotor displacement will exceed the limitation, which means a failure of operation. The existence of observer implies that $\mathrm{AOBC}$ has a lower robustness 
compared to $\mathrm{ABC}$ given the fact that average overshoot value and settling time are significantly larger in the simulation results for AOBC. That is because its feedback information is not accurate at first and it takes time for the observer to generate correct data.

\section{7 Comparison with PID}

In Figure 27 and Figure 28, we compare the simulation results of AOBC with that of PID. Both of the controllers have only position measurements. The PID gains are the optimized ones in Chapter III: $K_{p}=12258, K_{i}=2303, K_{d}=250$; the AOBC gains are the same as section IV.5. The solid line denotes the position displacement under the control of AOBC and the dotted line shows the PID control result.

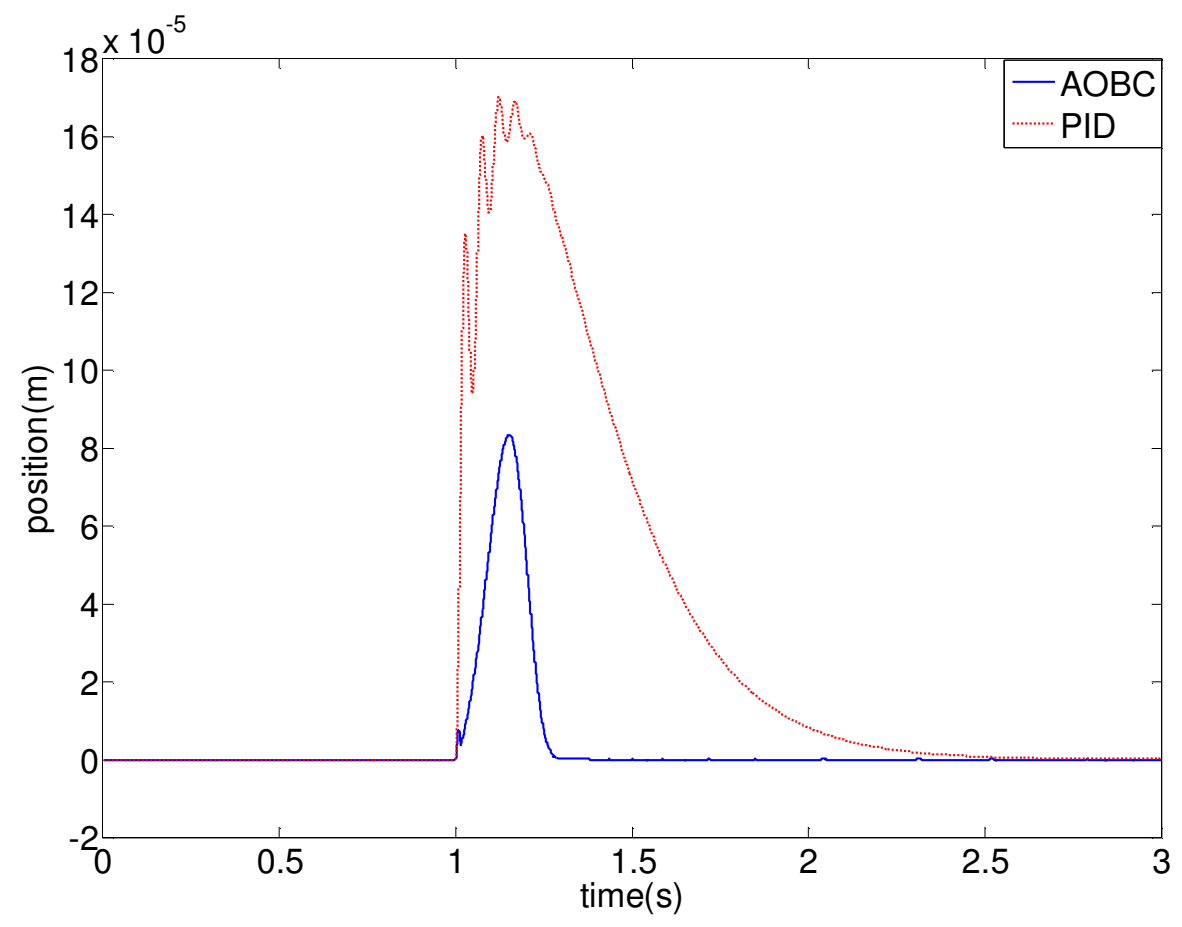

Figure 27: Position responses of $A O B C$ and PID controlled AMB systems 


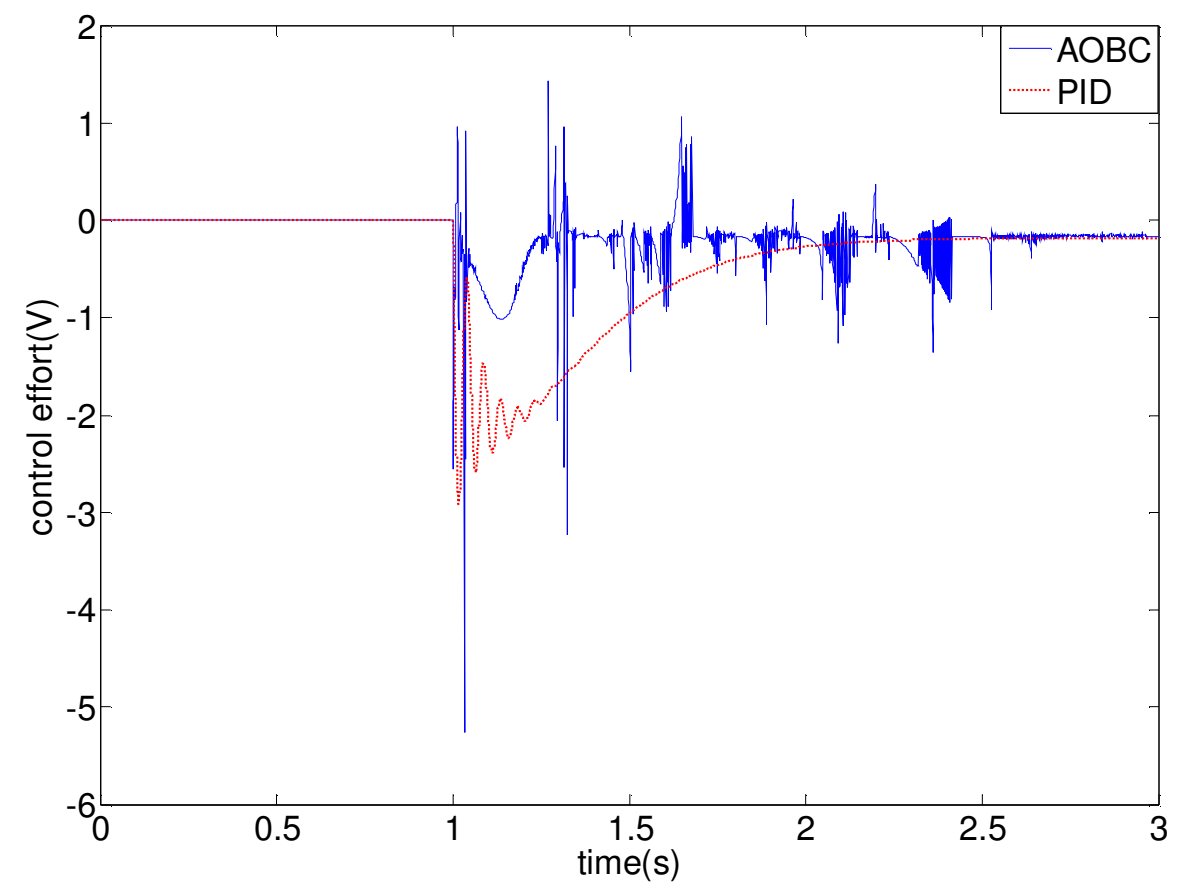

Figure 28: Control efforts of $A O B C$ and PID controlled AMB systems

As shown in Figure 27, the displacement response of the AOBC from equilibrium point is smaller than that of PID. Also, the settling time of AOBC is shorter than PID. However, Figure 28 shows that the control effort of AOBC has more oscillations than PID.

\section{8 Summary of the chapter}

In this chapter, because the only output is position displacement, an exponentially convergent observer is introduced in front of the AOBC to estimate the information of those non-measureable states. The control law is generated in a similar way as Chapter III with the addition of estimator error. Despite the facts that the states and uncertainty can be estimated and the system is forced to be stable, the simulation results show that the response of the closed-loop system is not as ideal as that of $\mathrm{ABC}$, and the robustness 
against either disturbance or parameter variations is weaker, too. On the other hand, AOBC requires more computation time due to the complexity of the control system. 


\section{CHAPTER V}

\section{SUMMARY AND FUTURE RESEARCH}

\section{1 Summary}

This thesis introduces two types of adaptive backstepping control, $\mathrm{ABC}$ and $\mathrm{AOBC}$, to regulate the AMB position in the presence of a force disturbance on its rotor. Our control objectives are to stabilize the closed-loop system and to discuss the tunings of the two types of adaptive controllers. A detailed process of how to construct the dynamic model of AMB was explained in the beginning. Lyapunov method had proved the stability of the control system without knowing the explicit knowledge of the solution. In simulation results, the effectiveness and robustness of the control system are verified, and $A B C$ and AOBC also estimate the value of the disturbance via adaptive updating law. Because the symbols used in design procedure represent the general case, $\mathrm{ABC}$ and $\mathrm{AOBC}$ methods are able to be applied to other nonlinear system models only if those systems are given as, 
or can be transformed, to the "strict feedback form" and "output feedback form" respectively.

In order to obtain better performances, we tuned the LCs and ACs separately to test their effects on the states, estimators and control effort in both ABC and AOBC. Simulations showed that increasing LCs could make response time, and the maximum overshoot smaller. Larger ACs drive the estimator to its target faster and also lead a smaller control effort. However, those increasing coefficients will lead to more oscillations in the transient response. Those experimental results could also be deduced from the physical meaning of the CLF's value: the increasing of the LCs will speed up the decrease of the system's energy by making $\dot{V}$ more negative. Though AOBC also successfully achieved the control goal, the overshoot value was larger than that in $\mathrm{ABC}$ with same settings. Also, the time that Simulink required for the simulation was longer due to the complexity of the controller.

Compared with PID, ABC and AOBC could achieve better performance if the Lyapunov coefficients' setting is proper. The overshoot was smaller and there were no oscillations in the transient response, and the settling time is shorter.

However, there are some shortcomings of AOBC. First of all, because an outputfeedback form is needed, AOBC is not applicable for those systems which can not be transformed to that form. Second, the speed of computing the adaptive control law is slow, which is a barrier in some situations in need of fast calculation, so this problem 
needs to be solved by a faster industrial computer. Finally, the control effort has an uneven trajectory when the Lyapunov coefficients are large, and we need to find a balanced way to tune the LCs and ACs to achieve both good response and low control effort.

\section{2 Future work}

In the future, a clear study of how to choose Lyapunov coefficients adequately should be made firstly since the experiments reveal that their variations influence the system performance to a large degree. We need to find a balanced way to set CLF coefficients and obtain a control law which could result in better outcomes than the best situation simulated above without sacrificing speed. Second, the observer-based ABC can be improved by employing different estimators which can provide more precise estimation information to minimize the observer errors' with which the control law deals.

As a promising advanced controller, adaptive backstepping has the potential to be widely applied in the real world with its reliability and the ability of on-line estimation of uncertainties. According to [13], there is a systematic way to design the control law, and how to apply this complicated control method to industry field needs to be considered. One possible way of implementation is to input the completed controller to the programmable control system, as long as the exact tuning method of the Lyapunov coefficients is discovered. Besides the programmable problem, the unsmooth control effort is also difficult to implement and needs to be improved. Another item is that the 
ignored conditions such as power loss and some equivalency assumptions should be handled by the robustness of the controller if no other method deals with that. 


\section{REFERENCES}

[1] S. C. Mukhopadhyay, C. Gooneratne and G. Sen Gupta "Magnetic bearing: an Integrated Platform for Teaching and Learning," in Proc. of $2^{\text {nd }}$ International Conference on Autonomous Robots and Agents, Palmerston North, New Zealand, Dec. 2004, pp. 283-288.

[2] Richard A.Rarick, Control of An Active Magnetic Beating with and without Position Sensing, Master's Thesis, Department of Electrical and Computer Engineering, Cleveland State University, Cleveland, OH, 2007.

[3] B. Polajžer, J. Ritonja, G. Štumberger, D. Dolinar and J.-P. Lecointe, "Decentralized PI/PD position control for active magnetic bearings," Electrical Engineering, vol. 89, no. 1 , pp. 53-59, Oct. 2006.

[4] John M. Oyster, On PID Controller Design for an Active Magnetic Bearing System, Bachelor's Thesis, Department of Electrical and Computer Engineering, Cleveland State University, Cleveland, OH, 2009.

[5] Timothy P. Denver, Gerald V. Brown, Ralph H Jansen, "Estimator Based Controller For High Speed Flywheel Magnetic Bearing System," NASA internal report (NASA/TM-2002-211795), Aug. 2002.

[6] Ha-Yong Kim, Chong-Won Lee, "Design and Control of Active Magnetic Bearing System with Lorentz Force-Type Axial Actuator," Mechatronics, vol. 16, no. 1, pp. 13-20, Feb. 2006. 
[7] Rafal Piotr Jastrzebski, Riku Pollanen, "Centralized Optimal Position Control for Active Magnetic Bearings: Comparison with Decentralized Control," Electrical Engineering, vol. 91, no. 2, pp. 101-114, Aug. 2009.

[8] Wojciech Grega, Adam Pilat, "Comparison of Linear Control Methods for an AMB system,” Int J Appl Math Comput Sci, vol. 15, no. 2, pp. 245-25, 2005.

[9] Ladislav Kucera, "Robustness of Self-Sensing Magnetic Bearing," in Proceedings of Magnetic Bearings Industrial Conference, Alexandria, Virginia, 1991, pp. 261270.

[10] Lichuan Li, Tadahiko Shinshi, and Akira Shimokohbe, "State Feedback Control for Active Magnetic Bearing Based on Current Change Rate Alone”, IEEE Transactions on Magnetics, vol. 40, no. 6, pp. 3512-3517, Nov. 2004.

[11] Myounggyu D. Noh, Eric H. Maslen, "Self-Sensing Magnetic Bearings Using Parameter Estimation," IEEE Transactions on Instrumentation and Measurement, vol. 46, no. 1, pp. 45-50, Feb. 1997.

[12] Baixu Su Alexander, Control of Active Magnetic Bearings For Flywheel Energy Storagy, Master's Thesis, Department of Electrical and Computer Engineering, Cleveland State University, Cleveland, OH, 2006.

[13] Miroslav Kristic, Ioannis Kanellakopoulos and Petar Kokotavic, Nonlinear and Adaptive Control Design, New York: Wiley-Interscience, 1995.

[14] Poemi Fernandez, Teodor Akinfiev, Manuel Armada, Climbing and Walking Robots, Springer Berlin Heidelberg, 2006. 
[15] Ö.Tolga Altmöz, "Adaptive Integral Backstepping Motion Control for Inverted Pendulum," International Journal of Computer and Information Science and Engineering, vol. 1, no. 4, pp. 192-195, 2007.

[16] A.Benaskeur, A. Desbiens, "Application of Adaptive Backstepping to the Stabilization of the Inverted Pendulum," in CCECE'98 Proceedings, Waterloo, Ontario, 1998, pp. 113-116.

[17] Jung-Hua Yang, Wen-Chun Hsu, "Adaptive backstepping control for electrically driven unmanned helicopter," Control Engineering Practice, vol. 17, no. 8, pp. 903913, Aug. 2009.

[18] R.R.Joshi, R.A.Gupta and A.K Wadhwani, "Adaptive Backstepping Controller Design and Implementation for a Matrix-Converter-Based IM Drive System," Journal of Theoretical and Applied Information Technology, vol. 3, no. 2, pp. 28-41, 2007.

[19] I. K. Boussehane, A. Hazzad, M. Rahli, B. Mazari and M. Kamli, "Mover Position Control of Linear Induction Motor Drive Using Adaptive Backstepping Controller with Integral Action,” Journal of Science and Engineering, vol. 12, no. 1, pp. 17-28, 2009.

[20] F. Farivar, M.A. Shoorehdeli, M. A. Nekoui and M. Teshnehlab, "Gaussian Radial Basis Adaptive Backstepping Control for a Class of Nonlinear System," Journal of Applied Science, vol. 9, no. 2, pp. 248-257, 2009.

[21] P. V. Kokotovic, "Joy of Feedback: Nonlinear and Adaptive," Control Systems Magazine, vol. 12, no. 3, pp. 7-17, June 1992. 
[22] Selim Sivrioglu, Kenzo Nonami, "Adaptive Output Backstepping Control of a Flywheel Zero-Bias AMB System with Parameter uncertainty," Nonlinear dynamics, vol. 48, no. 1-2, pp. 157-184, Apr. 2007.

[23] Mark W. Spong, Seth Hutchinso, M. Vidyasagar, Robot Modeling and Control, New Jersey John Wiley \& Sons Inc, 2006.

[24] Slotine J.J.E, Weiping Li, Applied Nonlinear System, Beijing, Chinese Mechanics Press, 2004 (in Chinese).

[25] Petros A. Ioannou, Jing Sun, Robust Adaptive Control, NewJersy, PTR PrenticeHall, 1996.

[26] G. Schweitzer, "Active Magnetic Bearings - Chances and Limitations," in Proceedings of the 6th International Conference on Rotor Dynamics, Sydney, Australia, Sep. 2002, pp. 1-14. 